\title{
Signaling Information Management in Entrepreneurial Firms' Financing Acquisition:
} An Integrated Signaling and Screening Perspective

\author{
Leven Jianwen Zheng, Xi' an Jiaotong-Liverpool University, China \\ Tao Bai, The University of Queensland, Australia \\ Adam R. Cross, Xi’an Jiaotong-Liverpool University, China
}

\begin{abstract}
Research has identified the significant effect of new ventures' signaling information management on their ability to secure private equity financing. This study adopts an integrated signaling and screening perspective to investigate investors' differing perceptions of signals from ventures across early financing stages. It proposes a three-step interpretation process. Based on an inductive multiple case study of signaler-receiver dyads, it finds that to reach a financing decision, angel investors extract a characteristic signal as the fundamental type, orchestrate an acting signal as a supplementary type, and scrutinize the consistency between both. However, venture capital investors extract an action signal as the fundamental type, orchestrate characteristic and endorsement signals as complementary types, and scrutinize the consistency among all three types.
\end{abstract}

\section{KEYWORDS}

Entrepreneurial Firms, Private Equity Financing, Screening Theory, Signaling Information Management, Signaling Theory

\section{INTRODUCTION}

Private equity financing is critical for new ventures to enhance their likelihood of survival and success, particularly in the early stages of their life cycle (Bruton et al., 2010; Cavallo et al., 2019; Pan et al., 2020; Tian et al., 2019). It is critical because new ventures often face the liability of "newness"; in general, they also have limited financial capital resources, which constrains their operations and development (Stinchcombe, 1965; Tian et al., 2019). However, new ventures can experience challenges when seeking to obtain financial support from private equity investors (Becker-Blease \& Sohl, 2015). One key reason for these challenges is that new ventures often face an information asymmetry problem caused by the lack of a credible operational track record (Fisher et al., 2016; Wang et al., 2019). In the absence of information regarding a venture's quality, entrepreneurial signaling information 
management have been identified as a key mechanism that enables new ventures to communicate their unobservable quality and potential value to investors, enabling them to secure private equity funding (e.g., Certo, 2003; Plummer et al., 2016).

Research based on signaling theory has revealed that new ventures make frequent use of multiple signal types in their pursuit of private equity financing (Drover et al., 2017; Vergne et al., 2018). These signals relate to several factors, such as the educational background and prior experience of entrepreneurs (e.g., Park et al., 2016); a venture's innovative product lines (e.g., Hoenig \& Henkel, 2015); and a venture's participation in strategic alliances (e.g., Pollock et al., 2010). However, the literature presents inconsistent findings regarding the effect of different signals on securing equity financing (Ko \& McKelvie, 2018). One important reason for these inconsistent findings is that studies have predominantly focused on new ventures as signalers to explore the effects of signals; it is generally assumed that signals are received and understood equally by all investors who are the signal receivers (Drover et al., 2017). However, financing decisions are made by an investor's determination of a signal's effectiveness (Bitektine, 2011), and investors' interpretation of signals may differ (Connelly et al., 2011). Moreover, different investor types, such as angel and venture capital (VC) investors, who have diverse priorities and focuses, may also interpret the same signal sent by ventures differently (Fisher et al., 2016). In summary, it is important to explore the process of how different equity investors interpret the signals sent by new ventures.

Therefore, to address knowledge gaps and further advance the new venture signaling literature, this study adopts an integrated signaling and screening framework to explore how different investors interpret the multiple types of signals from new ventures at different growth stages. Further, it uses data on signaler-receiver pairs in this framework. Adopting an inductive case study approach (Eisenhardt, 1989), the study investigates 16 new venture cases in the high-technology industry and their corresponding angel investors and venture capitalists. The high-technology industry provides a useful study context for two reasons. First, the complexity of high technology means that it is difficult for equity investors to assess new ventures; thus, information asymmetry is typically severe in this sector (Cannizzaro \& Weiner, 2018). Second, the development of technology-intensive products and services involves significant resource consumption and uncertainty, which means that securing external financing is often critical to a new venture's survival and development (Colombo \& Grilli, 2009; Gimmon \& Levie, 2010). Consequently, this research setting is ideal for examining the dynamic of multiple signals in the financing acquisition of new ventures.

Through multiple case studies, this study finds that investors tend to follow a three-step signal interpretation process to make investment decisions: first, they extract the fundamental signal; then, they orchestrate other signals to gain further understanding of a venture; and last, they scrutinize the consistency among signals. By focusing on two main types of private equity financing — angel and VC (Bruton et al., 2010) - this study concludes that angel investors extract the characteristic signal as the fundamental type, orchestrate the acting signal as a supplementary type, and scrutinize the consistency between these two signal types. VC investors extract the action signal as the fundamental type, orchestrate characteristic and endorsement signals as complementary types, and scrutinize the consistency among these three signal types.

This study provides several key theoretical contributions. First, it contributes to the literature on entrepreneurial financing by emphasizing the important role of receivers in interpreting the value of multiple signals sent by signalers (Connelly et al., 2011). Previous studies have mainly treated investors as passive (e.g., Hall, 2019; Ko \& McKelvie, 2018) and homogeneous (e.g., Plummer et al., 2016; Vaznyte \& Andries, 2019), and have paid insufficient attention to analyzing and distinguishing their diverse informational needs (Fisher et al., 2016). By developing the signal interpretation process framework, this study provides an in-depth understanding of the mechanism by which investors assess the signals sent by new ventures. Second, this study theorizes how investors evaluate new ventures through different signal combinations. The literature mostly assumes that different signal types work independently and that the combined effects of different signals are additive (Busenitz et al., 2005; 
Connelly et al., 2011). This study emphasizes the importance of supplementary and complementary signals in helping investors evaluate a focal venture. The interactions-not only the presence, but also the consistency - among different signal types provide a rich, complex view of the field and will advance the entrepreneurial signaling literature.

Last, by matching signalers and receivers across angel and VC financing stages to explore the effectiveness of signals, this study responds to calls for more in-depth, qualitative analyses of entrepreneurial ventures as they transition through their life cycle stages (e.g., Fisher et al., 2016). Studies on new venture signals have predominantly treated different investor types in the early financing stage in the same way; other studies have focused on one specific investor type-either angel investors (e.g., Ahlers et al., 2015) or venture capitalists (e.g., Hoenig \& Henkel, 2015; Hsu \& Ziedonis, 2013; Islam et al., 2018). Several studies have theoretically and empirically collapsed angel and VC financing into the aggregate "early-stage financing" concept (e.g., Plummer et al., 2016). In contrast, the present study distinguishes angels from VC investors and examines how these investors assess signals. Assessments can be viewed as quality assessments through the eyes of different types of investors (Fisher et al., 2017); thus, they are investor dependent (Bitektine, 2011).

The theoretical model developed in the next section is designed to integrate the signaling and screening perspectives. Section 3 explains the methodology for an inductive multiple case study of signaler-receiver dyads. Section 4 presents detailed findings from the case analysis. Last, Section 5 discusses the theoretical contributions and practical implications of this study and presents future research directions.

\section{THEORETICAL BACKGROUND}

\subsection{Signaling Theory}

Spence's signaling theory $(1973,2002)$ is widely employed in the entrepreneurial financing literature. In particular, this theory is used to explain how different signals can be used to reveal the value and quality of entrepreneurial firms through weakening information asymmetry between investors and firms. New ventures can then use signals to communicate their quality to investors-quality that would otherwise be challenging, if not impossible, for investors to discern for themselves (Plummer et al., 2016). The literature identifies various signals used by new ventures to communicate their unobservable quality to prospective investors and thus address the problem of information asymmetry (Certo, 2003).

To date, research on these various signals has predominantly concentrated on the stage at which an entrepreneurial firm undertakes an initial public offering (IPO) (e.g., Gomulya et al., 2019; Plummer et al., 2016). However, before this stage, new ventures often require two earlier "rounds" of financing to develop their business: an angel financing stage, followed by a VC financing stage (Bruton et al., 2009, 2010). While angel investors and venture capitalists are both a type of private equity investor, substantial differences exist between them (see Table 1). In turn, these may differentiate the evaluation of ventures, using the signals they value as indicators of quality (Bruton et al., 2010; Van Osnabrugge, 2000).

Importantly, and as signaling theory highlights, different receivers are likely to perceive and evaluate the same or similar signals from senders differently (Connelly et al., 2011). The literature strongly emphasizes the role and importance of signalers and their signals (e.g., Ko \& McKelvie, 2018), while neglecting the attention and interpretation of receivers-namely, potential investors (Drover et al., 2017). Thus, this literature seems to assume that different types of signals prompt equal and highly rational responses from all parties (Drover et al., 2017). Studies have generally viewed private equity investors as passive recipients of multiple signals, regarding them as a homogeneous group. This study argues that this view has created an incomplete understanding of how and why signaling unfolds as new ventures seek to acquire private equity financing. To address this limitation, the study 
Table 1. Contrasting signal audience attributes for angel investors and venture capitalists

\begin{tabular}{|l|l|l|}
\hline \multicolumn{1}{|c|}{ Signal Audience Attribute } & \multicolumn{1}{c|}{ Angel Investors } & \multicolumn{1}{c|}{ Venture Capitalists } \\
\hline Audience stages & Smaller; early stage; conception stage & $\begin{array}{l}\text { Larger; expansion stage; } \\
\text { commercialization stage }\end{array}$ \\
\hline Audience goals & $\begin{array}{l}\text { Longer-term financial and } \\
\text { non-financial returns; support } \\
\text { technological advancement; longer- } \\
\text { term investment horizon }\end{array}$ & $\begin{array}{l}\text { Short-term financial returns; exit the } \\
\text { investment in future at high multiples }\end{array}$ \\
\hline Audience norms & Longer-term financial self-interest & Shorter-term financial self-interest \\
\hline Audience values & Venture value creation & Portfolio value creation \\
\hline Audience control mechanism & $\begin{array}{l}\text { Behavior oriented; entrepreneur } \\
\text { behaviors; hands-on }\end{array}$ & $\begin{array}{l}\text { Outcome oriented; outcome of ventures; } \\
\text { strategic, often seeking a board seat }\end{array}$ \\
\hline
\end{tabular}

Source: Adapted from Bruton et al. (2010) and Fisher et al. (2016, 2017).

integrates a screening perspective with the signaling perspective to better capture the heterogeneity of signal receivers.

\subsection{An Integrated Signaling and Screening Framework}

When applying signaling theory, it is important to consider its counterpart—screening theory. The focus of screening theory moves from how signal senders convey multiple signals to how receivers prioritize and interpret multiple signal types (Gomulya \& Mishina, 2017). Hence, the signaling perspective focuses on how senders choose what signals to send, while the screening perspective emphasizes how signal receivers differentially assess the value of multiple signals (Gomulya \& Mishina, 2017; Sanders \& Boivie, 2004). Because of the processual nature of signaling, this study considers it important to integrate the signaling and screening perspectives into a single framework to understand the mutual needs of both new ventures and private equity investors. The screening perspective literature mainly provides explanations for how receivers process and interpret the value of a given isolated signal (e.g., Gomulya \& Mishina, 2017; Sanders \& Boivie, 2004). As a result, this literature provides little insight into how signal receivers perceive and interpret multiple signals during their evaluation process. Further, most studies consider receivers in isolation (Drover et al., 2017). However, since the evolution of a venture is, by definition, a dynamic process, it is necessary to explore how multiple signals may be perceived, as well as interpreted, by different receivers over time. Consequently, this study explores how multiple signals are perceived and interpreted across different financing stages during a new venture's early life cycle.

Moreover, signal screening studies highlight that the interpretation of particular signals can change owing to the nature of signal senders, as well as the signals themselves (Connelly et al., 2011), such as the different growth stage of a venture (Fisher et al., 2016), signal composition (Li \& McConomy, 2004) and interconnectedness among financing providers (Drover et al., 2017). Therefore, during a receiver's screening and evaluative process, a new venture's legitimacy and credibility will not have uniform effects on all the signals it transmits (Gomulya \& Mishina, 2017); that is, "the perceived trustworthiness of some signals is going to be more compromised than others" (Gomulya \& Mishina, 2017, p. 555). Based on screening theory arguments, some signals may be perceived and either interpreted or ignored, and investors may place greater emphasis on particular signals when making investment decisions about a focal venture. However, investor evaluation processes, and how they change across various early financing stages, remain understudied.

When combined, the signaling and screening perspectives focus attention on two main aspects to address the following research question: how does signal perception and interpretation evolve among signal receivers as they process multiple signal types across stages as a venture grows? First, it is 
necessary to understand investor perceptions of the different signal types sent by new ventures and to explore investor interpretations of these multiple signals as a whole, which implies that a broader understanding of a particular investor's evaluative process is required. Second, as new ventures often face different "rounds" of financing, it is important to employ a dynamic view to investigate the interpretations of different investors across the early stages of a venture. The signaling timeline involves two principal actors - the signaler and receiver-in addition to the signal itself (Connelly et al., 2011). Using this integrated signaling and screening conceptual framework, an explorative multiple case study of signaler-receiver dyads is used to investigate the signaling process, with a focus on how angel and VC investors interpret multiple signal types sent by new ventures across different early financing stages.

\section{RESEARCH METHODS}

\subsection{Sample Selection}

To address the research question, a case study method was adopted, using an inductive approach (Eisenhardt, 1989). Longitudinal case studies were conducted on 16 new high-technology ventures based in Beijing, People's Republic of China (henceforth "China"). Data were collected from new venture chief executive officers (CEOs) (i.e., signal senders), as well as from their corresponding angel investors and venture capitalists (i.e., signal receivers). Beijing was selected as the research setting because it is among the world's top five most active entrepreneurial environments; it also has the highest density of start-ups in China (Hemmert et al., 2019). In addition, China has generally witnessed a rapidly growing number of unicorns and new technology-intensive ventures over the past decade, with a vibrant VC industry (Yang \& Li, 2008; Zou et al., 2010). A high incidence and intensity of signal sender-receiver relationships are thus expected to exist there.

The case study examples were selected following a theoretical sampling procedure (Eisenhardt, 1989). To develop the sample selection criteria, approaches from the literature were adopted (e.g., Li \& Atuahene-Gima, 2001). In addition, interviews were conducted with experts, including two academic specialists in entrepreneurship, three leading angel investors, three major venture capitalists and 10 entrepreneurs from different industries and locations in China. This process led to the following sample selection criteria for the venture cases: (i) a top management team consisting of scientific experts or engineers; (ii) at least $30 \%$ of personnel being technical employees; (iii) at least 30\% expenditure on research and development (R\&D); and (iv) experience in securing private equity financing. Zhongguancun Science Park (ZSP) is one of the most established innovation parks in China (Li \& Atuahene-Gima, 2001) and it is the predominant location for new venture firms in Beijing (Hemmert et al., 2019). As such, lists of enterprises based at ZSP were obtained from the Zhongguancun Council and Beijing municipal government to build the sample. From these two lists, the CEOs of several high-technology ventures were selected at random and invited to participate in the study. In addition to confirming the above selection criteria, another condition was applied: that the principal investors in each new venture were willing to participate in the study. Following this process, 16 new ventures and their corresponding equity investors agreed to be involved: nine (Groups A, C, and E) based in ZSP and seven (Groups B, D, and F) located elsewhere in Beijing, with the latter included to conform with the study's theoretical sampling and to improve generalizability (Eisenhardt, 1989; Eisenhardt \& Graebner, 2007; Hallen \& Eisenhardt, 2012).

\subsection{Data Collection}

Data were collected from multiple sources (see Table 2): semi-structured interviews, archival records, websites, business plans and presentations on each of the 16 case study ventures, as well as from investor evaluation forms, notes, records, and feedback on a given venture. Adopting this approach 
allowed data triangulation to reduce bias and ensure data reliability, which bolstered the accuracy of the study's emergent theory (Eisenhardt, 1989; Hallen \& Eisenhardt, 2012; Yin, 1984; Zott \& Huy, 2007).

The primary data sources were entrepreneurs from the new ventures and their corresponding investors. The new venture interviewees were either the founder CEO or a co-founder (e.g., chief technology officers [CTO]) who had co-created the venture and could therefore share accurate insights into the venture's growth and financing history. Among investors, the person who had directly experienced a new venture's entire angel or VC financing process, and who had the authority to make a final decision about whether to invest in the new venture was interviewed. Overall, 142 face-to-face semi-structured interviews, each lasting 45 to 200 minutes, were conducted with the new venture CEOs, co-founders, and investors, mostly at the interviewees' work sites. In addition, one entrepreneur who had undertaken four entrepreneurial journeys and possessed extensive experience in both angel and VC financing acquisition, one top angel investor, and one top venture capitalist also participated in the interviews by asking questions and taking notes. These interviews with the multiple sources enabled the collection of a thick dataset and high-quality information from different perspectives.

The first round of semi-structured interviews was conducted from June to August 2017. By this time, most ventures had only acquired angel financing. The second round occurred from August to December 2018. By this time, many ventures had acquired subsequent rounds of financing. A semistructured interview approach was employed to conduct face-to-face interviews with entrepreneurs. Guidelines were established for interviews according to the research question. Interview questions were then refined by integrating feedback and suggestions from nine experts - three entrepreneurs, two entrepreneurial researchers, two top angel investors and two venture capitalists-in accordance with Van de Ven's (2007) recommendations. The interview schedule encompassed questions used to collect data about the individual entrepreneur (e.g., job title, job responsibility, role in venture financing activities), the venture and investor backgrounds and attributes (e.g., business line, industry focus) and financing activities (e.g., financing or investment, evaluative process). The entrepreneurs were invited to provide deep insights regarding what signals they used to attract angel and VC funds.

Informants from the investors were then asked to evaluate (using a 7-point scale) the importance of all signals they received in terms of influencing their funding decisions. They were then asked to describe and evaluate the process of how they perceived and interpreted the multiple signal information provided by the new ventures for their final investment decision making process. Open-ended questions were also asked to encourage CEOs and their co-founders to provide a comprehensive picture of the signals they sent, and to offer concrete illustrative examples of signals. Similarly, open-ended questions aimed to obtain more practical insights from investors, to understand their perceptions and interpretations of the information sent by the ventures' CEOs or co-founders.

\subsection{Data Analysis}

An inductive approach, along with a thematic analysis, was used to summarize the data information, identify patterns and constructs, and investigate relationships among the data (Berg et al., 2004; Eisenhardt \& Graebner, 2007; Gehman et al., 2018; M. B. Miles \& Huberman, 1994). To analyse the interviews and archival information, each individual case was first compiled (Aguinis \& Solarino, 2019; Eisenhardt, 1989), including information on the venture and its angel investor and venture capitalist, with a particular focus on identifying patterns of time-ordered events and their corresponding financing events. An individual case description was developed. This description stated how each new venture used different types of signals to acquire and achieve venture legitimacy to increase their likelihood of obtaining angel and VC funds, and to elucidate the process of how and why the corresponding investors interpreted different types of signals (Cassell \& Symon, 2004; Symon et al., 2018). The completed write-ups (both Chinese and English versions) were confirmed by the founder CEOs, co-founders and their corresponding investors. They were then reviewed and evaluated by industry and investment experts. Further, the case-replication method was followed for multiple data 
Table 2. Summary of data collection sources

\begin{tabular}{|c|c|c|c|c|c|c|c|c|c|c|}
\hline \multirow{3}{*}{\multicolumn{2}{|c|}{ Ventures }} & \multicolumn{9}{|c|}{ Data Sources } \\
\hline & & \multicolumn{4}{|c|}{ Interviews } & \multicolumn{2}{|c|}{ Documents } & \multicolumn{3}{|c|}{ Other Sources } \\
\hline & & Founder CEOs & Co-founders & AI & VC & Int. & Ext. & Inf. & Site visit & FB \\
\hline \multirow{4}{*}{ Group A } & ServiCo & 3 & 2 & 3 & 2 & 5 & 2 & 4 & 2 & 4 \\
\hline & TruckCo & 2 & 2 & 3 & 4 & 14 & 3 & 5 & 2 & 4 \\
\hline & EduCo & 2 & 2 & 3 & 2 & 8 & 1 & 16 & 2 & 4 \\
\hline & QTechCo & 4 & 2 & 4 & 3 & 6 & 3 & 5 & 2 & 4 \\
\hline \multirow{4}{*}{ Group B } & SoftCo & 3 & 0 & 2 & 3 & 6 & 3 & 5 & 2 & 4 \\
\hline & RobotCo & 3 & 1 & 3 & 4 & 5 & 6 & 11 & 2 & 4 \\
\hline & AICo & 3 & 0 & 3 & 3 & 7 & 2 & 2 & 2 & 4 \\
\hline & TestCo & 3 & 1 & 2 & 2 & 4 & 2 & 1 & 1 & 4 \\
\hline \multirow{3}{*}{ Group C } & SlockCo & 3 & 1 & 2 & 2 & 7 & 2 & 4 & 2 & 4 \\
\hline & ShuttleCo & 3 & 0 & 2 & 2 & 11 & 4 & 3 & 2 & 4 \\
\hline & MediCo & 2 & 1 & 2 & 4 & 15 & 3 & 2 & 2 & 4 \\
\hline \multirow{2}{*}{ Group D } & ChipCo & 3 & 2 & 3 & 2 & 8 & 1 & 1 & 2 & 4 \\
\hline & InterCo & 2 & 1 & 2 & 2 & 12 & 1 & 6 & 1 & 4 \\
\hline \multirow{2}{*}{ Group E } & VisionCo & 3 & 0 & 3 & 0 & 20 & 3 & 5 & 0 & 4 \\
\hline & ImageCo & 3 & 1 & 4 & 0 & 19 & 3 & 8 & 0 & 4 \\
\hline Group F & CarCo & 3 & 2 & 3 & 0 & 17 & 2 & 7 & 1 & 4 \\
\hline \multicolumn{2}{|l|}{ Sub-total } & 45 & 18 & 44 & 35 & - & - & - & - & - \\
\hline \multicolumn{2}{|l|}{ In total } & \multicolumn{4}{|l|}{142} & \multicolumn{5}{|l|}{-} \\
\hline
\end{tabular}

Note: $\mathrm{Al}=$ Angel investors; $\mathrm{VC}=$ Venture capitalists; Int. = Internal; Ext. = External; Inf. = Informal; FB = Feedback

analysis, which compared matched cases to confirm or refute emerging insights, gradually expanding the cross-case analysis (Kan et al., 2016; Strauss \& Corbin, 1998).

Based on two focus group discussions - one with five top angel investors and five entrepreneurs (Focus Group Discussion 1) and one with five venture capitalists and five entrepreneurs (Focus Group Discussion 2) - venture financing performance was designated as high (new venture achieved 5 million yuan or more in angel financing and 50 million yuan or more in VC financing); moderate (new venture achieved 2-5 million yuan in angel financing and 20-50 million yuan in VC financing); low (new venture achieved $\leq 2$ million yuan in angel financing and 10-20 million yuan in VC financing); or abandoned (new venture achieved no angel or VC financing). In addition to these focus group discussions, the ventures' average amount of financing for the threshold value was used to designate their financial performance, as indicated in Table 3.

For each case study, the process of data coding and exploration consisted of three important steps (Pratt, 2000, 2008). The first was open coding, the main aim of which was to better understand the signals that new ventures used to communicate with potential investors. Common statements identified in interviews and archival records formed the provisional categories coded as first-order constructs. For instance, several data segments associated with "educational level," "educational specialization," and "prior industry experience" were identified. After initial identification of the first-order constructs, the data content was further assessed and reviewed to refine them until no additional meaningful and discrete constructs were discovered. Axial coding was the second step, used to categorize and summarize the identified first-order constructs into more abstract and theoretical themes (Strauss \& 
Table 3. Private equity financing acquisition among sampled new ventures

\begin{tabular}{|c|c|c|c|c|c|c|}
\hline $\begin{array}{l}\text { Venture and } \\
\text { Growth Stage }\end{array}$ & $\begin{array}{l}\text { Approached } \\
\text { Potential } \\
\text { Investors }\end{array}$ & $\begin{array}{l}\text { Financing } \\
\text { Outcome }\end{array}$ & $\begin{array}{l}\text { Financing } \\
\text { Completion }\end{array}$ & $\begin{array}{l}\text { Venture } \\
\text { Status }\end{array}$ & $\begin{array}{c}\text { Financing } \\
\text { Amount } \\
\text { (yuan) }\end{array}$ & $\begin{array}{c}\text { Financing } \\
\text { Performance } \\
\text { Level }\end{array}$ \\
\hline \multicolumn{7}{|l|}{ Group A } \\
\hline \multicolumn{7}{|l|}{ ServiCo } \\
\hline Angel & $\begin{array}{l}2 \text { angel } \\
\text { investors }\end{array}$ & $\begin{array}{l}\text { Acquired } \\
\text { financing } \\
\text { from } 2 \text { angel } \\
\text { investors }\end{array}$ & $\bullet$ & $S$ & $8 \mathrm{M}$ & $\mathrm{H}$ \\
\hline $\mathrm{VC}$ & $2 \mathrm{VC}$ investors & $\begin{array}{l}\text { Acquired } \\
\text { financing from } \\
1 \text { VC investor }\end{array}$ & - & S & $60 \mathrm{M}$ & $\mathrm{H}$ \\
\hline \multicolumn{7}{|l|}{ TruckCo } \\
\hline Angel & $\begin{array}{l}6 \text { angel } \\
\text { investors }\end{array}$ & $\begin{array}{l}\text { Acquired } \\
\text { financing } \\
\text { from } 2 \text { angel } \\
\text { investors }\end{array}$ & $\bullet$ & $\mathrm{S}$ & $6 \mathrm{M}$ & $\mathrm{H}$ \\
\hline $\mathrm{VC}$ & $5 \mathrm{VC}$ investors & $\begin{array}{l}\text { Acquired } \\
\text { financing from } \\
2 \text { VC investors }\end{array}$ & - & $S$ & $120 \mathrm{M}$ & $\mathrm{H}$ \\
\hline \multicolumn{7}{|l|}{ EduCo } \\
\hline Angel & $\begin{array}{l}8 \text { angel } \\
\text { investors }\end{array}$ & $\begin{array}{l}\text { Acquired } \\
\text { financing } \\
\text { from } 2 \text { angel } \\
\text { investors }\end{array}$ & $\bullet$ & S & $4 \mathrm{M}$ & M \\
\hline $\mathrm{VC}$ & $20 \mathrm{VC}$ investors & $\begin{array}{l}\text { Acquired } \\
\text { financing from } \\
1 \text { VC investor }\end{array}$ & $\bullet$ & $\mathrm{S}$ & $90 \mathrm{M}$ & $\mathrm{H}$ \\
\hline \multicolumn{7}{|l|}{ QTechCo } \\
\hline Angel & $\begin{array}{l}10 \text { angel } \\
\text { investors }\end{array}$ & $\begin{array}{l}\text { Acquired } \\
\text { financing from } \\
1 \text { angel investor }\end{array}$ & $\bullet$ & S & $7 \mathrm{M}$ & $\mathrm{H}$ \\
\hline $\mathrm{VC}$ & $\begin{array}{l}20-30 \mathrm{VC} \\
\text { investors }\end{array}$ & $\begin{array}{l}\text { Acquired } \\
\text { financing from } \\
1 \mathrm{VC} \text { investor }\end{array}$ & $\bullet$ & S & $10.5 \mathrm{M}$ & $\mathrm{L}$ \\
\hline \multicolumn{7}{|l|}{ Group B } \\
\hline \multicolumn{7}{|l|}{ SoftCo } \\
\hline Angel & $\begin{array}{l}10-15 \text { angel } \\
\text { investors }\end{array}$ & $\begin{array}{l}\text { Acquired } \\
\text { financing } \\
\text { from } 1 \text { angel } \\
\text { investors }\end{array}$ & $\bullet$ & S & $2 \mathrm{M}$ & $\mathrm{L}$ \\
\hline $\mathrm{VC}$ & $\begin{array}{l}15-20 \mathrm{VC} \\
\text { investors }\end{array}$ & $\begin{array}{l}\text { Acquired } \\
\text { financing from } \\
2 \mathrm{VC} \text { investors }\end{array}$ & $\bullet$ & S & $40 \mathrm{M}$ & M \\
\hline
\end{tabular}


Table 3. Continued

\begin{tabular}{|c|c|c|c|c|c|c|}
\hline $\begin{array}{l}\text { Venture and } \\
\text { Growth Stage }\end{array}$ & $\begin{array}{l}\text { Approached } \\
\text { Potential } \\
\text { Investors }\end{array}$ & $\begin{array}{l}\text { Financing } \\
\text { Outcome }\end{array}$ & $\begin{array}{l}\text { Financing } \\
\text { Completion }\end{array}$ & $\begin{array}{l}\text { Venture } \\
\text { Status }\end{array}$ & $\begin{array}{c}\text { Financing } \\
\text { Amount } \\
\text { (yuan) }\end{array}$ & $\begin{array}{c}\text { Financing } \\
\text { Performance } \\
\text { Level }\end{array}$ \\
\hline \multicolumn{7}{|l|}{ RobotCo } \\
\hline Angel & $\begin{array}{l}7 \text { angel } \\
\text { investors }\end{array}$ & $\begin{array}{l}\text { Acquired } \\
\text { financing from } \\
1 \text { angel investor }\end{array}$ & $\bullet$ & S & $3 \mathrm{M}$ & M \\
\hline $\mathrm{VC}$ & $6 \mathrm{VC}$ investors & $\begin{array}{l}\text { Acquired } \\
\text { financing from } \\
2 \mathrm{VC} \text { investors }\end{array}$ & $\bullet$ & S & $60.5 \mathrm{M}$ & $\mathrm{H}$ \\
\hline \multicolumn{7}{|l|}{ AICo } \\
\hline Angel & $\begin{array}{l}5-10 \text { angel } \\
\text { investors }\end{array}$ & $\begin{array}{l}\text { Acquired } \\
\text { financing } \\
\text { from } 2 \text { angel } \\
\text { investors }\end{array}$ & $\bullet$ & $S$ & $5.5 \mathrm{M}$ & $\mathrm{H}$ \\
\hline $\mathrm{VC}$ & $5 \mathrm{VC}$ investors & $\begin{array}{l}\text { Acquired } \\
\text { financing from } \\
2 \mathrm{VC} \text { investors }\end{array}$ & $\bullet$ & $S$ & $30 \mathrm{M}$ & M \\
\hline \multicolumn{7}{|l|}{ TestCo } \\
\hline Angel & $\begin{array}{l}14 \text { angel } \\
\text { investors }\end{array}$ & $\begin{array}{l}\text { Acquired } \\
\text { financing } \\
\text { from } 2 \text { angel } \\
\text { investors }\end{array}$ & $\bullet$ & $S$ & $4 \mathrm{M}$ & M \\
\hline $\mathrm{VC}$ & $17 \mathrm{VC}$ investors & $\begin{array}{l}\text { Acquired } \\
\text { financing from } \\
2 \mathrm{VC} \text { investors }\end{array}$ & $\bullet$ & S & $60 \mathrm{M}$ & $\mathrm{H}$ \\
\hline \multicolumn{7}{|l|}{ Group C } \\
\hline \multicolumn{7}{|l|}{ SlockCo } \\
\hline Angel & $\begin{array}{l}7 \text { angel } \\
\text { investors }\end{array}$ & $\begin{array}{l}\text { Acquired } \\
\text { financing from } \\
2 \text { angels }\end{array}$ & $\bullet$ & S & $5 \mathrm{M}$ & $\mathrm{H}$ \\
\hline $\mathrm{VC}$ & $\begin{array}{l}30-40 \mathrm{VC} \\
\text { investors }\end{array}$ & $\begin{array}{l}\text { Did NOT } \\
\text { successfully } \\
\text { acquire VC } \\
\text { financing }\end{array}$ & $\mathrm{O}$ & $\mathrm{D}$ & - & $\mathrm{A}$ \\
\hline \multicolumn{7}{|l|}{ ShuttleCo } \\
\hline Angel & $\begin{array}{l}10-15 \text { angel } \\
\text { investors }\end{array}$ & $\begin{array}{l}\text { Acquired } \\
\text { financing from } \\
2 \text { angels }\end{array}$ & $\bullet$ & S & $6 \mathrm{M}$ & $\mathrm{H}$ \\
\hline $\mathrm{VC}$ & $\begin{array}{l}20-30 \mathrm{VC} \\
\text { investors }\end{array}$ & $\begin{array}{l}\text { Did NOT } \\
\text { successfully } \\
\text { acquire VC } \\
\text { financing }\end{array}$ & $\mathrm{O}$ & $\mathrm{D}$ & - & A \\
\hline
\end{tabular}


Table 3. Continued

\begin{tabular}{|c|c|c|c|c|c|c|}
\hline $\begin{array}{l}\text { Venture and } \\
\text { Growth Stage }\end{array}$ & $\begin{array}{l}\text { Approached } \\
\text { Potential } \\
\text { Investors }\end{array}$ & $\begin{array}{l}\text { Financing } \\
\text { Outcome }\end{array}$ & $\begin{array}{l}\text { Financing } \\
\text { Completion }\end{array}$ & $\begin{array}{l}\text { Venture } \\
\text { Status }\end{array}$ & $\begin{array}{c}\text { Financing } \\
\text { Amount } \\
\text { (yuan) }\end{array}$ & $\begin{array}{c}\text { Financing } \\
\text { Performance } \\
\text { Level }\end{array}$ \\
\hline \multicolumn{7}{|l|}{ MediCo } \\
\hline Angel & $\begin{array}{l}3 \text { angel } \\
\text { investors }\end{array}$ & $\begin{array}{l}\text { Acquired } \\
\text { financing from } \\
1 \text { angel }\end{array}$ & $\bullet$ & $S$ & $4 \mathrm{M}$ & M \\
\hline $\mathrm{VC}$ & $\begin{array}{l}\text { Around } 25 \mathrm{VC} \\
\text { investors }\end{array}$ & $\begin{array}{l}\text { Did NOT } \\
\text { successfully } \\
\text { acquire VC } \\
\text { financing }\end{array}$ & $\mathrm{O}$ & $\mathrm{D}$ & - & A \\
\hline \multicolumn{7}{|l|}{ Group D } \\
\hline \multicolumn{7}{|l|}{ ChipCo } \\
\hline Angel & $\begin{array}{l}8 \text { angel } \\
\text { investors }\end{array}$ & $\begin{array}{l}\text { Acquired financing } \\
\text { from } 3 \text { angels }\end{array}$ & $\bullet$ & S & $7.5 \mathrm{M}$ & $\mathrm{H}$ \\
\hline $\mathrm{VC}$ & $\begin{array}{l}\text { Around } 30 \mathrm{VC} \\
\text { investors }\end{array}$ & $\begin{array}{l}\text { Did NOT } \\
\text { successfully } \\
\text { acquire VC } \\
\text { financing }\end{array}$ & $\mathrm{O}$ & D & - & A \\
\hline \multicolumn{7}{|l|}{ InterCo } \\
\hline Angel & $\begin{array}{l}12 \text { angel } \\
\text { investors }\end{array}$ & $\begin{array}{l}\text { Acquired } \\
\text { financing from } \\
2 \text { angels }\end{array}$ & $\bullet$ & $S$ & $1 \mathrm{M}$ & Low \\
\hline $\mathrm{VC}$ & $\begin{array}{l}\text { Around } 50 \mathrm{VC} \\
\text { investors }\end{array}$ & $\begin{array}{l}\text { Did NOT } \\
\text { successfully } \\
\text { acquire VC } \\
\text { financing }\end{array}$ & $\mathrm{O}$ & D & - & A \\
\hline \multicolumn{7}{|l|}{ Group E } \\
\hline \multicolumn{7}{|l|}{ VisionCo } \\
\hline Angel & $\begin{array}{l}\text { Around } 60 \\
\text { angel investors }\end{array}$ & $\begin{array}{l}\text { Did NOT } \\
\text { successfully } \\
\text { acquire angel } \\
\text { financing }\end{array}$ & $\mathrm{O}$ & $\mathrm{D}$ & - & A \\
\hline No VC & - & - & - & - & - & - \\
\hline \multicolumn{7}{|l|}{ ImageCo } \\
\hline Angel & $\begin{array}{l}\text { Around } 50 \\
\text { angel investors }\end{array}$ & $\begin{array}{l}\text { Did NOT } \\
\text { successfully } \\
\text { acquire angel } \\
\text { financing }\end{array}$ & $\mathrm{O}$ & $\mathrm{D}$ & - & A \\
\hline NO VC & - & - & - & - & - & - \\
\hline \multicolumn{7}{|l|}{ Group F } \\
\hline \multicolumn{7}{|l|}{ CarCo } \\
\hline $\begin{array}{l}\text { Angel } \\
\text { NO VC }\end{array}$ & $\begin{array}{l}\text { Around } 40 \\
\text { angel investors } \\
\text { - }\end{array}$ & $\begin{array}{l}\text { Did NOT } \\
\text { successfully } \\
\text { acquire angel } \\
\text { financing } \\
\text { - }\end{array}$ & $\begin{array}{l}\mathrm{O} \\
-\end{array}$ & $\mathrm{D}$ & - & $\begin{array}{l}\text { A } \\
-\end{array}$ \\
\hline
\end{tabular}

Note: $\bullet=$ completed and successfully acquired financing; $\mathrm{O}=$ completed and failed to acquire financing; $\mathrm{S}=$ survived; $\mathrm{D}=\operatorname{died} ; \mathrm{H}=$ high; $\mathrm{M}=$ moderate; $\mathrm{L}=\operatorname{low} ; \mathrm{A}=$ abandoned 
Corbin, 1998). For example, a second-order theme of "educational background" was developed to consolidate the first-order constructs of "educational level" and "educational specialization." The identified second-order themes are consistent with the literature on entrepreneurial financing (e.g., Gomulya et al., 2019; Plummer et al., 2016). The second-order themes were further developed and categorized into several aggregated dimensions. After developing the aggregated theoretical themes, they were compared with the literature to confirm if they provided a comprehensive understanding of the corresponding literature. For instance, "educational background" and "industry experience" were conflated into "signaler characteristics."

\section{FINDINGS}

Case study analysis findings are summarized based on the study's processual signaling and screening framework. First, all main signals sent by the new ventures are identified, and then how investors observed and interpreted the signals they received are reported.

\subsection{Signal Transmission}

Research has shown that entrepreneurs send multiple signal types as a way to communicate their ventures' quality, improving the likelihood of financing acquisition (Plummer et al., 2016). In keeping with this argument, the sampled ventures in this study were found to typically transmit three types of signals - characteristic, action, and endorsement signals - to communicate venture quality to potential investors, as shown in Table 4 (see Appendix A for detailed information).

\subsubsection{Signaler Characteristics}

When seeking both angel and venture capitalist financing, each of the 16 new ventures proactively sent characteristic signals to their targeted investors. In particular, these characteristics related to educational background, prior R\&D experience, prior industrial experience, management experience, entrepreneurial experience, and the joint working experience of the founding team (Plummer et al., 2016). For instance, the founder of TruckCo clarified that the "characteristics of the founding team were the core part" of the signals communicated when they acquired angel funds. Similarly, the founder CEO and co-founder CTO of RobotCo each stated that "the characteristics of the founding team were essential" for both angel and VC financing acquisition. For investors, value could be estimated by considering a series of venture features (Stuart et al., 1999). The most common characteristics that outside investors used to evaluate the quality of a new venture were those that reflect the strength of its human capital (Beckman et al., 2007).

\subsubsection{Signaler Actions}

The actions that ventures undertake in entrepreneurial activities can reveal the value of these ventures to potential investors (Rindova et al., 2005). Interviews with the 16 ventures revealed that actions specifically relate to the possession of patents, the development of prototypes, and the introduction of products or services (or both) onto the market. For example, the founder CEO and co-founder CTO of TestCo stated that they explained the "new products and the applicational scenarios of their products" to targeted venture capitalists because they believed that these new products would most effectively define "their technological advancement," "innovative capability," or "commercial viability" for potential investors. Similarly, in relation to acquiring VC financing, the founder CEO of AICo observed that "specific visible action, particularly prototypes and new product" could set a firm "apart from competitors." 
Table 4. A summary of different types of signals sent by new ventures

\begin{tabular}{|c|c|c|c|c|c|c|c|c|c|c|c|c|c|c|c|}
\hline \multirow[b]{3}{*}{$\begin{array}{c}\text { New } \\
\text { Ventures } \\
\text { and Stages }\end{array}$} & \multicolumn{15}{|c|}{ Signals } \\
\hline & \multicolumn{7}{|c|}{ Characteristics } & \multicolumn{3}{|c|}{ Actions } & \multicolumn{5}{|c|}{ Endorsements } \\
\hline & 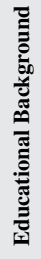 & 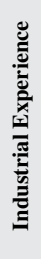 & 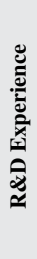 & 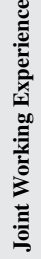 & 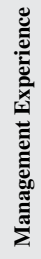 & 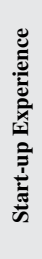 & 立 & है & है & 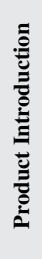 & 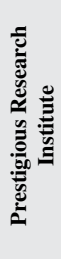 & 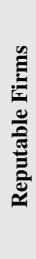 & 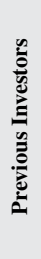 & 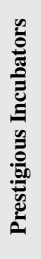 & $\begin{array}{l}\text { है } \\
\text { है } \\
\text { है } \\
\text { है }\end{array}$ \\
\hline \multicolumn{16}{|l|}{ ServiCo } \\
\hline Angel & $\bullet$ & $\bullet$ & $\bullet$ & $\bullet$ & $\mathrm{O}$ & $\bullet$ & $\mathrm{O}$ & $\mathrm{O}$ & $\mathrm{O}$ & $\mathrm{O}$ & • & • & $\mathrm{O}$ & $\bullet$ & $\mathrm{O}$ \\
\hline $\mathrm{VC}$ & $\bullet$ & $\bullet$ & $\bullet$ & $\bullet$ & $\mathrm{O}$ & $\bullet$ & $\mathrm{O}$ & $\mathrm{O}$ & $\mathrm{O}$ & $\bullet$ & $\bullet$ & $\bullet$ & $\bullet$ & $\bullet$ & $\mathrm{O}$ \\
\hline \multicolumn{16}{|l|}{ TruckCo } \\
\hline Angel & $\bullet$ & $\bullet$ & $\bullet$ & $\mathrm{O}$ & $\bullet$ & $\bullet$ & $\bullet$ & $\mathrm{O}$ & $\mathrm{O}$ & $\mathrm{O}$ & • & $\bullet$ & $\mathrm{O}$ & - & $\mathrm{O}$ \\
\hline $\mathrm{VC}$ & $\bullet$ & $\bullet$ & $\bullet$ & $\mathrm{O}$ & • & $\bullet$ & $\bullet$ & $\bullet$ & - & $\mathrm{O}$ & • & • & $\bullet$ & $\bullet$ & - \\
\hline \multicolumn{16}{|l|}{$\underline{\text { EduCo }}$} \\
\hline Angel & $\bullet$ & $\bullet$ & $\bullet$ & $\bullet$ & $\bullet$ & $\mathrm{O}$ & $\bullet$ & $\bullet$ & • & $\mathrm{O}$ & - & $\mathrm{O}$ & $\mathrm{O}$ & $\mathrm{O}$ & $\mathrm{O}$ \\
\hline $\mathrm{VC}$ & $\bullet$ & $\bullet$ & $\bullet$ & $\bullet$ & $\bullet$ & $\mathrm{O}$ & $\bullet$ & $\bullet$ & • & $\bullet$ & $\bullet$ & $\bullet$ & $\bullet$ & $\mathrm{O}$ & - \\
\hline \multicolumn{16}{|l|}{ QTechCo } \\
\hline Angel & $\bullet$ & $\bullet$ & $\bullet$ & $\bullet$ & $\bullet$ & $\mathrm{O}$ & $\mathrm{O}$ & $\mathrm{O}$ & $\mathrm{O}$ & $\mathrm{O}$ & - & $\mathrm{O}$ & $\mathrm{O}$ & $\bullet$ & $\mathrm{O}$ \\
\hline $\mathrm{VC}$ & $\bullet$ & $\bullet$ & $\bullet$ & $\bullet$ & $\bullet$ & $\mathrm{O}$ & $\mathrm{O}$ & $\bullet$ & $\bullet$ & $\bullet$ & $\mathrm{O}$ & $\bullet$ & $\bullet$ & $\mathrm{O}$ & $\mathrm{O}$ \\
\hline \multicolumn{16}{|l|}{$\underline{\text { SoftCo }}$} \\
\hline Angel & $\bullet$ & $\bullet$ & $\bullet$ & $\mathrm{O}$ & $\mathrm{O}$ & $\bullet$ & $\mathrm{O}$ & $\mathrm{O}$ & • & $\mathrm{O}$ & $\mathrm{O}$ & $\mathrm{O}$ & $\mathrm{O}$ & $\bullet$ & $\mathrm{O}$ \\
\hline $\mathrm{VC}$ & $\bullet$ & $\bullet$ & $\bullet$ & $\mathrm{O}$ & $\mathrm{O}$ & $\bullet$ & $\mathrm{O}$ & $\mathrm{O}$ & $\mathrm{O}$ & $\bullet$ & $\mathrm{O}$ & • & $\bullet$ & $\bullet$ & - \\
\hline \multicolumn{16}{|l|}{$\underline{\text { RobotCo }}$} \\
\hline Angel & $\bullet$ & $\bullet$ & $\bullet$ & $\bullet$ & $\bullet$ & $\mathrm{O}$ & $\mathrm{O}$ & - & - & $\mathrm{O}$ & $\bullet$ & $\mathrm{O}$ & $\mathrm{O}$ & $\mathrm{O}$ & $\mathrm{O}$ \\
\hline $\mathrm{VC}$ & $\bullet$ & $\bullet$ & $\bullet$ & $\bullet$ & • & $\mathrm{O}$ & $\mathrm{O}$ & - & • & $\bullet$ & • & • & $\bullet$ & $\mathrm{O}$ & $\mathrm{O}$ \\
\hline \multicolumn{16}{|l|}{$\underline{\text { AICo }}$} \\
\hline Angel & $\bullet$ & $\bullet$ & $\bullet$ & $\bullet$ & $\bullet$ & $\mathrm{O}$ & $\bullet$ & $\mathrm{O}$ & • & $\mathrm{O}$ & $\mathrm{O}$ & $\mathrm{O}$ & $\mathrm{O}$ & $\mathrm{O}$ & $\mathrm{O}$ \\
\hline $\mathrm{VC}$ & $\bullet$ & $\bullet$ & $\bullet$ & $\bullet$ & • & $\mathrm{O}$ & $\bullet$ & $\bullet$ & • & $\bullet$ & $\mathrm{O}$ & $\bullet$ & $\bullet$ & $\mathrm{O}$ & $\mathrm{O}$ \\
\hline \multicolumn{16}{|l|}{$\underline{\text { TestCo }}$} \\
\hline Angel & $\bullet$ & $\bullet$ & $\bullet$ & $\mathrm{O}$ & • & $\bullet$ & $\mathrm{O}$ & $\mathrm{O}$ & • & $\mathrm{O}$ & $\mathrm{O}$ & $\mathrm{O}$ & $\mathrm{O}$ & $\mathrm{O}$ & $\mathrm{O}$ \\
\hline $\mathrm{VC}$ & $\bullet$ & $\bullet$ & $\bullet$ & $\mathrm{O}$ & • & $\bullet$ & $\mathrm{O}$ & $\mathrm{O}$ & $\mathrm{O}$ & $\bullet$ & $\mathrm{O}$ & $\mathrm{O}$ & $\bullet$ & $\mathrm{O}$ & $\mathrm{O}$ \\
\hline \multicolumn{16}{|l|}{$\underline{\text { SlockCo }}$} \\
\hline Angel & $\bullet$ & $\bullet$ & $\mathrm{O}$ & $\bullet$ & $\mathrm{O}$ & $\bullet$ & $\mathrm{O}$ & $\mathrm{O}$ & - & $\mathrm{O}$ & $\mathrm{O}$ & $\mathrm{O}$ & $\mathrm{O}$ & $\mathrm{O}$ & $\mathrm{O}$ \\
\hline $\mathrm{VC}$ & $\bullet$ & $\bullet$ & $\mathrm{O}$ & $\bullet$ & $\mathrm{O}$ & $\bullet$ & $\mathrm{O}$ & $\mathrm{O}$ & $\bullet$ & $\bullet$ & $\mathrm{O}$ & $\mathrm{O}$ & $\bullet$ & $\mathrm{O}$ & $\mathrm{O}$ \\
\hline \multicolumn{16}{|l|}{ ShuttleCo } \\
\hline Angel & $\bullet$ & $\bullet$ & • & $\mathrm{O}$ & $\bullet$ & $\bullet$ & $\mathrm{O}$ & $\mathrm{O}$ & $\mathrm{O}$ & $\mathrm{O}$ & $\bullet$ & $\bullet$ & $\mathrm{O}$ & $\bullet$ & $\bullet$ \\
\hline $\mathrm{VC}$ & $\bullet$ & $\bullet$ & • & $\mathrm{O}$ & $\bullet$ & $\bullet$ & $\bullet$ & $\bullet$ & • & $\mathrm{O}$ & • & $\bullet$ & $\bullet$ & $\bullet$ & • \\
\hline \multicolumn{16}{|l|}{$\underline{\text { MediCo }}$} \\
\hline Angel & $\bullet$ & • & • & • & • & $\mathrm{O}$ & $\mathrm{O}$ & $\mathrm{O}$ & • & $\mathrm{O}$ & $\mathrm{O}$ & $\mathrm{O}$ & $\mathrm{O}$ & $\mathrm{O}$ & $\mathrm{O}$ \\
\hline
\end{tabular}




\begin{tabular}{|c|c|c|c|c|c|c|c|c|c|c|c|c|c|c|c|}
\hline \multirow[b]{3}{*}{$\begin{array}{c}\text { New } \\
\text { Ventures } \\
\text { and Stages }\end{array}$} & \multicolumn{15}{|c|}{ Signals } \\
\hline & \multicolumn{7}{|c|}{ Characteristics } & \multicolumn{3}{|c|}{ Actions } & \multicolumn{5}{|c|}{ Endorsements } \\
\hline & 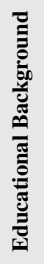 & 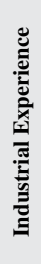 & 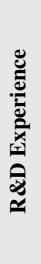 & 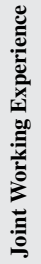 & 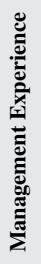 & 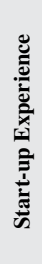 & 竞 & है & 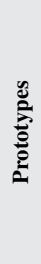 & 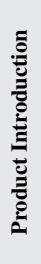 & 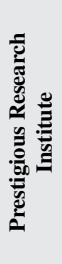 & 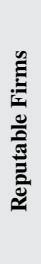 & 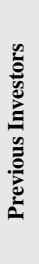 & 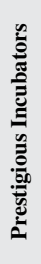 & 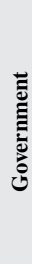 \\
\hline $\mathrm{VC}$ & $\bullet$ & • & $\bullet$ & $\bullet$ & $\bullet$ & $\mathrm{O}$ & $\mathrm{O}$ & $\mathrm{O}$ & $\bullet$ & $\bullet$ & $\mathrm{O}$ & $\mathrm{O}$ & $\bullet$ & $\mathrm{O}$ & $\mathrm{O}$ \\
\hline \multicolumn{16}{|l|}{ ChipCo } \\
\hline Angel & • & • & $\bullet$ & $\bullet$ & $\mathrm{O}$ & $\mathrm{O}$ & $\mathrm{O}$ & $\mathrm{O}$ & $\mathrm{O}$ & $\mathrm{O}$ & $\bullet$ & $\bullet$ & $\mathrm{O}$ & $\mathrm{O}$ & $\bullet$ \\
\hline $\mathrm{VC}$ & • & • & $\bullet$ & $\bullet$ & $\mathrm{O}$ & $\mathrm{O}$ & $\bullet$ & $\bullet$ & • & $\bullet$ & $\mathrm{O}$ & $\mathrm{O}$ & $\bullet$ & $\mathrm{O}$ & $\bullet$ \\
\hline \multicolumn{16}{|l|}{$\underline{\text { InterCo }}$} \\
\hline Angel & - & - & $\bullet$ & $\mathrm{O}$ & $\bullet$ & $\mathrm{O}$ & $\mathrm{O}$ & $\mathrm{O}$ & $\bullet$ & $\mathrm{O}$ & $\mathrm{O}$ & $\mathrm{O}$ & $\mathrm{O}$ & $\mathrm{O}$ & $\mathrm{O}$ \\
\hline $\mathrm{VC}$ & • & • & $\bullet$ & $\mathrm{O}$ & $\bullet$ & $\mathrm{O}$ & $\mathrm{O}$ & $\mathrm{O}$ & $\bullet$ & $\mathrm{O}$ & $\mathrm{O}$ & $\bullet$ & $\bullet$ & $\mathrm{O}$ & $\mathrm{O}$ \\
\hline \multicolumn{16}{|l|}{ VisionCo } \\
\hline Angel & • & - & $\bullet$ & $\mathrm{O}$ & $\mathrm{O}$ & $\mathrm{O}$ & $\mathrm{O}$ & $\mathrm{O}$ & • & $\mathrm{O}$ & - & $\mathrm{O}$ & $\mathrm{O}$ & $\mathrm{O}$ & $\mathrm{O}$ \\
\hline \multicolumn{16}{|l|}{ ImageCo } \\
\hline Angel & • & • & $\bullet$ & $\mathrm{O}$ & $\mathrm{O}$ & $\mathrm{O}$ & $\mathrm{O}$ & $\mathrm{O}$ & • & $\mathrm{O}$ & $\mathrm{O}$ & $\mathrm{O}$ & $\mathrm{O}$ & $\mathrm{O}$ & • \\
\hline \multicolumn{16}{|l|}{ CarCo } \\
\hline Angel & • & • & $\mathrm{O}$ & $\bullet$ & $\mathrm{O}$ & • & $\mathrm{O}$ & $\mathrm{O}$ & • & $\mathrm{O}$ & $\mathrm{O}$ & $\mathrm{O}$ & $\mathrm{O}$ & $\mathrm{O}$ & $\mathrm{O}$ \\
\hline
\end{tabular}

Note: $\bullet=$ signal present in sending process; $\mathrm{O}=$ no such signal

\subsubsection{Signaler Endorsements}

In addition to signaling their characteristics and actions, the case ventures sent endorsement signals to communicate the quality of their venture to targeted investors. One important type of endorsement signal identified in the study relates to third-party affiliations, and particularly to being associated with prestigious research institutions and universities, governments, and reputable firms. For instance, as the founder of ServiCo explained, "when we showed our venture to targeted venture capitalists, we used about three pages of PowerPoint slides to introduce and highlight our current strategic cooperation with two global top 500 enterprises." Similarly, when TruckCo approached an angel investor for financing, the CEO "showed and described the venture's cooperation agreements with some Chinese listed firms to investors."

Studies have shown that such third-party affiliations can enhance the market valuation of ventures (Gomulya et al., 2019; Plummer et al., 2016) and facilitate new venture growth (Eisenhardt \& Schoonhoven, 1996; Song et al., 2016). These outcomes may be possible because external stakeholders may regard these affiliations as independent evaluations of a venture's potential value and quality (Plummer et al., 2016), and the third party may provide a venture with resources (i.e., information, knowledge, supplies) and enhanced competitive capability (McEvily \& Zaheer, 1999). Therefore, at the early financing stage, third-party affiliations can serve as an endorsement signal that conveys information to investors about the prospects of a new venture. These affiliations can also communicate that third-party actors may help a venture enhance its future prospects by contributing valuable resources (Petkova, 2012; Pollock et al., 2010; Pollock \& Gulati, 2007). 


\subsection{Signal Screening}

Having identified the signals sent by new ventures, this study will now outline the corresponding investors and consider how they observed and perceived the signals received from their new venture. The findings are reported in Table 5. To better understand the mechanisms of interpreting signals, this study proposes that investors follow an interpretative process; they: (i) extract signal information and decide which type of signal is most fundamental; (ii) orchestrate other signals with the fundamental signal to further understand the venture; and (iii) scrutinize the content consistency of these signals to make the final funding decision.

\subsection{Extraction}

\subsubsection{Extraction by Angel Investors}

At the angel financing stage, all investors interviewed considered the characteristics of the founding team to be the fundamental signal that enabled them to evaluate the unobservable quality of a new venture. For instance, the angel investor for ServiCo (AngelServiCo) explained that "the most important assessment was their team, their founder and co-founders, [because] this helped us to understand the depth and breadth of their knowledge and why they could conduct the start-up." To explain why they invested in TruckCo, the angel investor explained that "we invested in [TruckCo]... because of their venture team. They are experts in the area... The most important criteria must be the quality of the [founding] team."

This study identifies several underlying reasons for why angel investors consider founding team characteristics the fundamental signal that influenced their decision making. One of the most important processes angel investors must employ is evaluating the existing technological and learning capabilities of the founding team. Characteristics such as educational level and educational specialization provide angel investors with evidence that they can use to assess the knowledge base, skills accumulation, and learning capabilities of the founding team. For example, as AngelServiCo described:

The start-up [ServiCo] is [a] technology-oriented, or product-oriented business, [so] their educational degrees and majors are very important. We evaluated their core team members' knowledge bases and learning capabilities, especially the CEO and CTO. [This is because] at the beginning of running a start-up is a process of constant learning.

Similarly, AngelTruckCo explained:

Educational background and working experience are the two most important indicators that can reflect their team members' qualifications. More importantly, [from this] we believed that this team (the founding team of TruckCo) has [a] deep understanding in their area, [since] I believe their research papers, their PhD degrees, [and] R\&D experience were good evidence and certification.

In contrast, three ventures among the case studies were rejected by their targeted angel investors, who noted that the ventures' "resource bases" or "team capabilities" did not convince them to invest. For instance, AngelVisionCo explained that both the CEO and CTO "lacked accumulation in the [business] field," which was "a really important foundation for such a high-technology start-up."

Another important assessment from angel investors relates to the teamwork capability of the founding team. In this study, angel investors often had to evaluate the teamwork abilities of founding team members and, specifically, of the founder CEO, the CTO, and the chief operational officer (COO). In particular, the signals used to assess these teamwork and collaboration capabilities referred to the founding team members' joint working and start-up experience. This was because these characteristics could help angel investors evaluate "whether the founding team members [of AICo] 
Table 5. Signals observed and interpreted by angels and venture capitalists

\begin{tabular}{|c|c|c|c|c|c|c|c|c|c|c|c|}
\hline \multicolumn{4}{|c|}{$\begin{array}{l}\text { Signal senders sent multiple signals to signal receivers } \\
\text { (Signal sending process) }\end{array}$} & \multicolumn{8}{|c|}{$\begin{array}{l}\text { Signal receivers interpreted multiple signals they received from signal } \\
\text { senders (Signal sense-making process) }\end{array}$} \\
\hline \multirow[b]{2}{*}{$\begin{array}{l}\text { Ventures and } \\
\text { Growth Stages }\end{array}$} & \multicolumn{3}{|c|}{ Signal } & \multirow[b]{2}{*}{$\begin{array}{c}\text { Investors across } \\
\text { different growth } \\
\text { stages }\end{array}$} & \multicolumn{3}{|c|}{$\begin{array}{l}\text { Fundamental or } \\
\text { Elementary Signal }\end{array}$} & \multicolumn{3}{|c|}{$\begin{array}{c}\text { Supplementary } \\
\text { or } \\
\text { Complementary } \\
\text { Signal }\end{array}$} & \multirow{2}{*}{ 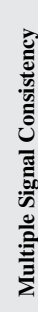 } \\
\hline & 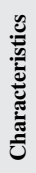 & : & 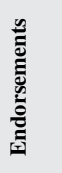 & & 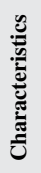 & 农 & 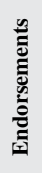 & 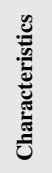 & : & 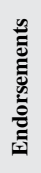 & \\
\hline \multicolumn{12}{|l|}{$\underline{\text { ServiCo }}$} \\
\hline Angel & $\bullet$ & $\mathrm{O}$ & $\mathrm{O}$ & AngelServiCo & $\bullet$ & $\mathrm{O}$ & $\mathrm{O}$ & $\mathrm{O}$ & $\bullet$ & $\mathrm{O}$ & $\square$ \\
\hline $\mathrm{VC}$ & - & • & - & VCServiCo & $\mathrm{O}$ & - & $\mathrm{O}$ & - & $\mathrm{O}$ & $\bullet$ & $\mathbf{\square}$ \\
\hline \multicolumn{12}{|l|}{ TruckCo } \\
\hline Angel & $\bullet$ & $\mathrm{O}$ & $\bullet$ & AngelTruckCo & $\bullet$ & $\mathrm{O}$ & $\mathrm{O}$ & $\mathrm{O}$ & $\mathrm{O}$ & $\mathrm{O}$ & $\square$ \\
\hline $\mathrm{VC}$ & $\bullet$ & • & $\bullet$ & VCTruckCo & $\mathrm{O}$ & $\bullet$ & $\mathrm{O}$ & $\bullet$ & $\mathrm{O}$ & $\bullet$ & $\mathbf{\square}$ \\
\hline \multicolumn{12}{|l|}{$\underline{\text { EduCo }}$} \\
\hline Angel & $\bullet$ & • & $\mathrm{O}$ & AngelEduCo & $\bullet$ & $\mathrm{O}$ & $\mathrm{O}$ & $\mathrm{O}$ & $\bullet$ & $\mathrm{O}$ & 口 \\
\hline $\mathrm{VC}$ & $\bullet$ & • & - & VCEduCo & $\mathrm{O}$ & $\bullet$ & $\mathrm{O}$ & $\bullet$ & $\mathrm{O}$ & $\bullet$ & 口 \\
\hline \multicolumn{12}{|l|}{ QTechCo } \\
\hline Angel & $\bullet$ & $\mathrm{O}$ & $\bullet$ & AngelQTechCo & $\bullet$ & $\mathrm{O}$ & $\mathrm{O}$ & $\mathrm{O}$ & $\mathrm{O}$ & $\mathrm{O}$ & $\square$ \\
\hline VC & $\bullet$ & • & $\bullet$ & VCQTechCo & $\mathrm{O}$ & $\bullet$ & $\mathrm{O}$ & $\bullet$ & $\mathrm{O}$ & $\bullet$ & $\mathbf{\square}$ \\
\hline \multicolumn{12}{|l|}{$\underline{\text { SoftCo }}$} \\
\hline Angel & $\bullet$ & • & $\bullet$ & AngelSoftCo & $\bullet$ & $\mathrm{O}$ & $\mathrm{O}$ & $\mathrm{O}$ & $\bullet$ & $\mathrm{O}$ & $\mathbf{\square}$ \\
\hline $\mathrm{VC}$ & $\bullet$ & • & - & VCSoftCo & $\mathrm{O}$ & • & $\mathrm{O}$ & $\bullet$ & $\mathrm{O}$ & $\bullet$ & $\mathbf{\square}$ \\
\hline \multicolumn{12}{|l|}{ RobotCo } \\
\hline Angel & $\bullet$ & • & - & AngelRobotCo & $\bullet$ & $\mathrm{O}$ & $\mathrm{O}$ & $\mathrm{O}$ & $\bullet$ & $\mathrm{O}$ & $\mathbf{\square}$ \\
\hline $\mathrm{VC}$ & - & • & $\bullet$ & VCRobotCo & $\mathrm{O}$ & $\bullet$ & $\mathrm{O}$ & $\bullet$ & $\mathrm{O}$ & $\bullet$ & $\mathbf{\square}$ \\
\hline \multicolumn{12}{|l|}{ AICo } \\
\hline Angel & $\bullet$ & • & - & AngelAICo & $\bullet$ & $\mathrm{O}$ & $\mathrm{O}$ & $\mathrm{O}$ & $\bullet$ & $\mathrm{O}$ & $\mathbf{\square}$ \\
\hline $\mathrm{VC}$ & $\bullet$ & • & - & VCAICo & $\mathrm{O}$ & $\bullet$ & $\mathrm{O}$ & $\bullet$ & $\mathrm{O}$ & $\bullet$ & $\mathbf{\square}$ \\
\hline \multicolumn{12}{|l|}{ TestCo } \\
\hline Angel & $\bullet$ & • & $\mathrm{O}$ & AngelTestCo & $\mathrm{O}$ & $\mathrm{O}$ & $\mathrm{O}$ & $\mathrm{O}$ & $\bullet$ & $\mathrm{O}$ & 口 \\
\hline $\mathrm{VC}$ & $\bullet$ & • & $\mathrm{O}$ & VCTestCo & $\mathrm{O}$ & $\bullet$ & $\mathrm{O}$ & $\bullet$ & $\mathrm{O}$ & $\bullet$ & $\mathbf{\square}$ \\
\hline \multicolumn{12}{|l|}{$\underline{\text { SlockCo }}$} \\
\hline Angel & $\bullet$ & • & $\mathrm{O}$ & AngelSlockCo & $\bullet$ & $\mathrm{O}$ & $\mathrm{O}$ & $\mathrm{O}$ & $\bullet$ & $\mathrm{O}$ & $\mathbf{\square}$ \\
\hline $\mathrm{VC}$ & $\bullet$ & • & $\bullet$ & VCSlockCo & $\mathrm{O}$ & $\bullet$ & $\mathrm{O}$ & • & $\mathrm{O}$ & $\bullet$ & 口 \\
\hline \multicolumn{12}{|l|}{ ShuttleCo } \\
\hline Angel & $\bullet$ & $\mathrm{O}$ & - & AngelShuttleCo & $\bullet$ & $\mathrm{O}$ & $\mathrm{O}$ & $\mathrm{O}$ & $\mathrm{O}$ & $\mathrm{O}$ & $\square$ \\
\hline
\end{tabular}




\begin{tabular}{|c|c|c|c|c|c|c|c|c|c|c|c|}
\hline \multicolumn{4}{|c|}{$\begin{array}{l}\text { Signal senders sent multiple signals to signal receivers } \\
\text { (Signal sending process) }\end{array}$} & \multicolumn{8}{|c|}{$\begin{array}{l}\text { Signal receivers interpreted multiple signals they received from signal } \\
\text { senders (Signal sense-making process) }\end{array}$} \\
\hline \multirow[b]{2}{*}{$\begin{array}{l}\text { Ventures and } \\
\text { Growth Stages }\end{array}$} & \multicolumn{3}{|c|}{ Signal } & \multirow[b]{2}{*}{$\begin{array}{l}\text { Investors across } \\
\text { different growth } \\
\text { stages }\end{array}$} & \multicolumn{3}{|c|}{$\begin{array}{l}\text { Fundamental or } \\
\text { Elementary Signal }\end{array}$} & \multicolumn{3}{|c|}{$\begin{array}{c}\text { Supplementary } \\
\text { or } \\
\text { Complementary } \\
\text { Signal }\end{array}$} & \multirow{2}{*}{ 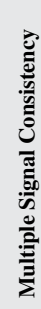 } \\
\hline & 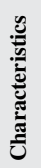 & 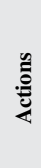 & 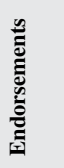 & & 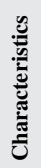 & : & 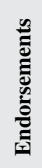 & 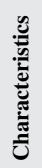 & 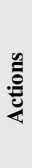 & 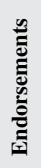 & \\
\hline $\mathrm{VC}$ & $\bullet$ & $\bullet$ & - & VCShuttleCo & $\mathrm{O}$ & - & $\mathrm{O}$ & $\bullet$ & $\mathrm{O}$ & $\bullet$ & $\mathbf{\square}$ \\
\hline \multicolumn{12}{|l|}{ MediCo } \\
\hline Angel & $\bullet$ & $\bullet$ & $\mathrm{O}$ & AngelMediCo & $\bullet$ & $\mathrm{O}$ & $\mathrm{O}$ & $\mathrm{O}$ & - & $\mathrm{O}$ & $\mathbf{\square}$ \\
\hline $\mathrm{VC}$ & $\bullet$ & $\bullet$ & $\bullet$ & VCMediCo & $\mathrm{O}$ & - & $\mathrm{O}$ & $\bullet$ & $\mathrm{O}$ & $\bullet$ & $\mathbf{\square}$ \\
\hline \multicolumn{12}{|l|}{ ChipCo } \\
\hline Angel & $\bullet$ & $\mathrm{O}$ & $\bullet$ & AngelChipCo & $\bullet$ & $\mathrm{O}$ & $\mathrm{O}$ & $\mathrm{O}$ & $\bullet$ & $\mathrm{O}$ & $\square$ \\
\hline $\mathrm{VC}$ & $\bullet$ & $\bullet$ & & VCChipCo & $\mathrm{O}$ & - & $\mathrm{O}$ & $\bullet$ & $\mathrm{O}$ & $\bullet$ & $\mathbf{\square}$ \\
\hline \multicolumn{12}{|l|}{ InterCo } \\
\hline Angel & $\bullet$ & $\bullet$ & $\mathrm{O}$ & AngelInterCo & $\bullet$ & $\mathrm{O}$ & $\mathrm{O}$ & $\mathrm{O}$ & $\mathrm{O}$ & $\mathrm{O}$ & $\mathbf{\square}$ \\
\hline $\mathrm{VC}$ & $\bullet$ & $\bullet$ & $\bullet$ & VCInterCo & $\mathrm{O}$ & $\bullet$ & $\mathrm{O}$ & $\bullet$ & $\mathrm{O}$ & $\bullet$ & $\mathbf{\square}$ \\
\hline \multicolumn{12}{|l|}{$\underline{\text { VisionCo }}$} \\
\hline Angel & $\bullet$ & $\bullet$ & • & AngelVisionCo & $\bullet$ & $\mathrm{O}$ & $\mathrm{O}$ & $\mathrm{O}$ & - & $\mathrm{O}$ & $\mathbf{\square}$ \\
\hline ImageCo & & & & & $\bullet$ & & & & - & & \\
\hline Angel & $\bullet$ & $\bullet$ & $\bullet$ & AngelImageCo & $\bullet$ & $\mathrm{O}$ & $\mathrm{O}$ & $\mathrm{O}$ & $\bullet$ & $\mathrm{O}$ & $\mathbf{\square}$ \\
\hline$\underline{\text { CarCo }}$ & & & & & $\bullet$ & & & & & & \\
\hline Angel & $\bullet$ & - & $\mathrm{O}$ & AngelCarCo & • & $\mathrm{O}$ & $\mathrm{O}$ & $\mathrm{O}$ & $\mathrm{O}$ & $\mathrm{O}$ & $\square$ \\
\hline
\end{tabular}

Note: $\bullet=$ this type of signal present in sending and interpreting process; $\circ=$ no such type of signal; $\square=$ investors did not check and interpret signal consistency between fundamental and supplementary signals; $\mathbf{-}=$ investors did not check and interpret signal consistency between fundamental and supplementary signals or elementary and complementary signals

have [the] capability to effectively work together to better identify the best business opportunities in the field" (AngelAICo) and to ascertain "if they [the founding team of RobotCo] could reduce team conflicts and were capable of avoiding inferior business opportunities, which have very limited latent potential" (AngelRobotCo). Specifically, when commenting on the quality of the founding team members' prior joint working and start-up experience, and their relevance to TruckCo's business scope, AngelTruckCo stated:

Their prior joint working experience was really important... I believed that the TruckCo has a very good team composition, the core founding team members have worked together for more than five years, rather than teams of close, like-minded individuals... And the core team is made up of members with diverse skills... As an independent and new start-up, their teaming capability matters... especially for the early stage of the venture and [the] sustainable development [of the venture].

Similarly, AngelEduCo emphasized the investment logic used to assess the characteristics of venture EduCo's founding team, noting: 
The prior joint working experience of the CEO and CTO as well as [the] start-up experience of the $\mathrm{COO}$ enables us to assess the founding team's collaboration capability associated with freeing up time and limited resources, adapting to technology and environment change, and avoiding serious missteps in the initial stage of running a business.

In contrast, a significant reason for the angel investor rejections of the VisionCo, ImageCo, and CarCo ventures was that their existing technological and learning and team capabilities were unconvincing. Each of the angel investors involved emphasized "weaknesses," or a "lack" of "resource bases/accumulations," or "collaboration capabilities" as key reasons for not investing in these businesses.

In addition, founding team characteristics, such as educational background and joint working experience, provided historical indicators for angel investors to track the origin of the ventures' entrepreneurial concepts or idea development. In the study sample, angel investors revealed that they attempt to "track" and "understand" the "origin" of the ventures' entrepreneurial concepts, based on the founding teams' characteristics. For example, AngelServiCo and AngelSoftCo interpreted the characteristics of the new ventures' CEOs, CTOs, and COOs, and explained that they tried to track their "original ideas" and "the developmental history of those ideas" by investigating and evaluating the founder CEOs' and co-founders' experience and background. Further, AngelVisionCo explained that they "had difficulties [tracking] the origins of venture VisionCo's entrepreneurial concepts based on the founding team's background, so we rejected their invitation."

\subsubsection{Venture Capitalist Extraction}

In relation to the VC financing stage, the fundamental assessments of all venture capitalists were focused on the type of action signals that related to whether the ventures had introduced new products into markets - to demonstrate their innovation and commercialization capabilities and the potential market need for their products. For example, VCTruckCo stated that "our top priority is the demonstrations and products of it (TruckCo)"; VCAICo explained their investment logic by saying that "the actual action [of the venture] is an essential"; VCServiCo noted that "definitely, the status of product was a relatively important indicator, among others."

Analysis of the information provided by venture informants, and the interpretations from their corresponding VC investors, identified several mechanisms to explain why VC investors considered action signals to be fundamental, and therefore used these to evaluate a venture's unobservable quality and value. First, VC investors tended to assess the ventures' commercialization potential in transferring innovative capabilities and technologies into high-technology products. All venture capitalists used the terms "commercialization capability" or "technology transfer capability" to explain why they considered action signals the key basis for their evaluation. For example, VCTruckCo explained their interpretation of TruckCo's innovative actions as follows:

The venture (TruckCo) had shown their executive and productive capabilities to produce visible outcomes (products)... From their past and current innovative activities and visible outcomes, we could see they focused on the ability to create and use advanced technologies in [the] automotive driving area, and did not excessively emphasize... the technologies themselves... They had transferred their unsubstituted technologies and capabilities into visible and commercial automotive trucks... Even though it was the first-generation automotive trucks, based on our analysis on their outcomes, we invested [in] it without any hesitation.

Second, the VC investors tended to evaluate and predict a product's potential market need. Venture capitalists used the term 'the true market need' to highlight their focus on ventures. For example, VCServiCo emphasized: 
The venture (ServiCo) had demonstrated its products to us... [and] drawn the potential market shares and returns of its products to us... We checked the sales data of its products for our reference to see if they had already introduced products into markets and analyzed the market situation of their products... [and] of course, checked the market feedback [on the products].

Similarly, VCAICo noted:

Products are the combination of technologies and markets. Products not only can reflect how the techniques develop them, but also can reveal how the products fit the market demands. We consequently analyzed the status of... [ServiCo's] products to evaluate if their intelligent ovens matched the real needs of the market... [and] the potential growth ability [of products].

\subsection{Orchestration}

\subsubsection{Angel Orchestration}

Based on the multiple signals sent by all ventures to their corresponding angel investors, and these investors' interpretations of these signals, it was determined that founding team characteristics were the only signals used by angel investors to make their investment decision for two main reasons. One was that the angel investors' priority was to evaluate the perceived human risks of the team. That is, they sought to investigate whether the founding team was qualified enough to run a new venture and was able to perform this task reliably. As AngelServiCo observed:

The team is the DNA of the venture... Everything is about the team at this stage. [Therefore], we used much time to check the DNA of each founder and co-founder of [venture ServiCo], [their] majors in school, prior working experience, particularly their personality, technology capability and industry experience... we found... (ServiCo) had a really good team DNA, [and] high-quality people. We trusted them. We trusted they can do this [business]... This was enough for us to make our investment decision.

Similarly, AngelRobotCo revealed:

What impressed me most was the CEO, CTO, and another co-founder, [the] CMO [chief marketing officer] [of RobotCo]. This team has very strong technology background and R\&D capability... They have passion. When they introduced their business logic, I saw the full... passion and energy. They worked for Huawei together before... They came together to run Robot for a common purpose... to develop products that can help and change the world... I would say the CEO [and] the team touched us... importantly, [it] helped us reduce uncertainty and increased our investment confidence... We only took a couple of days to make our [investment] decision.

Another important reason for the weight given to founding team characteristics was that angel investors needed to evaluate the rationale and feasibility of a new venture's business logic. In the study sample, founding team characteristics enabled angel investors to answer why and how the founding team would be able to run their proposed business successfully. All angel investors said that they answered these two questions before they invested in the venture. AngelTestCo explained:

When we went to visit their office site, their (TestCo's) CEO used lots of time to introduce their demonstrations [of products], [and] new products to us... To be honest, at that moment, we did not really care about their products and demonstrations. It did not matter what they (products) were... what kept reverberating in my mind was, how they came to this point (business), why [it] was them, 
why they could run it... [Thus], I asked the CEO and co-founders many questions about their background... to view their histories and development tracks.

AngelQTechCo stated that they invested in QTechCo because the "background of the founding team convinced me to believe them... I believe they can run their start-up logically and successfully." Similarly, AngelTruckCo explained: "we are more concerned about their team, the quality of their team, we needed to understand how they understood the development direction and trend of the industry, what innovative things they could [contribute] to the industry and society."

At the angel financing stage, angel investors considered the ventures' actions a supplementary signal in relation to making investment decisions. According to the information from both founders and angel investors, a supplementary signal can be defined as additional signals in the angel financing context. For example, AngelServiCo mentioned that they considered patents, product demonstrations, and product introductions as "credit-adding items." AngelRobotCo clarified that "it did not matter, even though they did not have patents and products at that very early stage." Two important reasons were given for why angel investors considered actions supplementary signals. One was that those actions provided some supplementary evidence for angel investors to confirm the technological and learning capability, as well as the team capability, of the founding team. For example, AngelEduCo explained that "[EduCo's] patents and demonstrations of products gave some additional information for us to confirm our assessment conclusions of their founding team... [and] to confirm their technology and team collaboration capability." Another reason was that new ventures had their own pace of innovation. Each angel investor asserted that they understood and respected that innovation had its own pace; thus, they considered action signals supplementary. For example, AngelTruckCo explained that TruckCo intended to "create a new thing, an innovative truck in the world. Innovative things always need time, particularly at the beginning stage of innovations." Even though TruckCo did not have patents, demonstrations, or products, AngelTruckCo invested a large amount of money in this venture in the hope that TruckCo "could take enough time to get a deeper understanding of their technology, industry and their business logics at this very early stage... A real innovation cannot be rushed." Further, AngelRobotCo stated that "it [does] not matter... [If] they [make] relatively slow progress on their technology development."

\subsubsection{Venture Capitalist Orchestration}

According to $\mathrm{VC}$ investor interpretations, it was found that the characteristics, actions, and endorsements of signalers formed an integrative orchestration: all elements were important criteria for assessing legitimacy at the VC financing stage. Based on the information provided by both VC investors and founder CEOs, characteristic signals and endorsement signals were deemed complementary. A complementary signal is defined as a type of signal that must be interpreted along with other types to indicate its quality to investors. In this case, it could be understood that action signals completed or balanced characteristic and endorsement signals to reveal the quality of action signals and of the venture. For example, VCTruckCo explained:

We... deeply evaluated... [TruckCo's] founding team... demonstrations... products... [and] cooperation agreements with others (organizations)... If it had problems in one of these dimensions, it probably would be confronted with challenges... even failures... We would not invest in it.

This also occurred in the other case studies. For example, VCTestCo noted that when they evaluated TestCo, they combined characteristics, actions, and endorsements as "an inseparable integrity"; VCRobotCo stated that these three dimensions "were mutually related, inter-crossed, mutually restrictive... mutually promoted."

Two main reasons lay behind the VC investor evaluation of founding team characteristics as complementary signals. One was that VC investors needed to assess the team's product-iterative 
capabilities, which were interpreted as a critical influence on the ventures' market value. For example, VCEduCo described their evaluation of EduCo in the following way:

Even though EduCo's products had been introduced into markets... [and] over 40 patents had been granted at that stage... for a new start-up company, at that stage... they had to keep iterating their current products... and to integrate their patents, their technologies into future product developments... [Therefore], we evaluated the characteristics of the team to evaluate [its] potential quality... and capability... to see if the team could iterate fast enough to develop the right products to fit the real market needs before they run out money.

Another reason was that VC investors needed to evaluate the ventures' operational capabilities, which were also interpreted as having a critical influence on the ventures' market value. In all cases, VC investors clarified that operational capabilities-including management, promotional, and production capabilities-were "much more important" at the VC than the angel stage. For example, VC INVESTORSoftCo described their quality evaluations in these terms:

We did evaluate [the] characteristics of the team, [and] we noticed that the team had strong capability to develop their venture from start-up to scale-up... [and] had capability to duplicate and expand their products into different application scenarios... for example... production capability and market expansion capability.

VC investors also needed to evaluate the ventures' affiliations with other organizations, because the combination of a venture's actions and affiliations helped VC investors assess the ventures' market value more precisely from an outsider perspective. For example, when VCRobotCo evaluated the market value and quality of RobotCo, they deliberately investigated the contract agreements for RobotCo's affiliations, which provided them with more information to evaluate the venture's real market value: "we checked the contents of RobotCo's affiliation contracts very carefully... meanwhile, we also contacted their affiliated partners for the purpose of due diligence." Therefore, VCRobotCo concluded that understanding RobotCo's affiliations enabled them to determine the real "uncertainties" and "risks" regarding the venture's products and market value. Conversely, VC investors tried to evaluate the real value of a venture's affiliations with third parties. Since ventures were still in the very early stages of their life cycle, they were often constrained by resource limitations. Thus, VC investors had to understand how a venture could strategically acquire valuable external resources to accelerate new product development. For example, VCAICo mentioned that "through our investigation and evaluation, we were happy to see that its (AICo's) affiliations with [two reputable enterprises in China] provided it with additional distribution channels... and useful market feedback."

\subsection{Scrutinizing}

After scanning all signals that they received and scrutinizing the relationships between them, investors in the study sample further analyzed the detailed content of these signals. A key feature of signal content was whether it was consistent across signals; content consistency influenced signal credibility and reliability (Connelly et al., 2011).

\subsubsection{Angel Scrutinizing}

At the angel financing stage, there was typically a need for angel investors to evaluate the consistency between characteristics and action signals. In all cases, angel investors evaluated the consistency between founding team characteristics and granted patents and prototypes of the products. These evaluations helped them weigh the perceived human risks of the ventures more precisely. All angel investors strongly contended that the "connection" or "continuity" between the "founders' 
backgrounds" and their "undertaken actions" could help reduce "uncertainty" or enhance their "confidence" to invest. For example, AngelInterCo noted that "the essential connection between the founders' industrial background and their working products act[ed] as an enabler for our investment in InterCo." In contrast, AngelVisionCo AngelCarCo and AngelImageCo identified the "loose connection," "non-background-driven" and "illogical" connections between founders' characteristics and working products as reasons to not invest.

The angel investors emphasized consistency between characteristics and actions for two main reasons. The first was that they evaluated the continuity direction of ventures. Here, based on the evaluation information provided by angel investors, continuity direction is defined as a continuous connection between a venture's undertaken actions (e.g., products) and its founding team's characteristics (e.g., founders' industrial experience). Specifically, evaluating consistency between a founder's characteristics and their undertaken actions enabled angel investors to determine whether product creation and development were continuously reflected in the founder's characteristics, including their industrial experience, R\&D experience, and educational background. With SlockCo, AngelSlockCo revealed that "as an angel investor, I felt very fortunate and excited to meet such founding team. They insisted on their own tracks and tried to identify some potential opportunities and to make them come true." Similarly, AngelSTestCo stated: "I believed they can do something in their field, since they stay on their own ground by their continuous efforts." In addition, as AngelServiCo explained:

After I heard the experiences and histories of the CEO [of ServiCo] and his co-founders, I had a much clearer conception [of] their business logic, and I can then link their product design and creation to the characteristics they mentioned to me.

In contrast, AngelVisionCo identified a "direction shift" and AngelCarCo asserted that "the founders were not experts in the field of their main products" as reasons for rejecting the venture. As AngelImageCo explained:

We did not find a specific connection between the founders' previous background and their current ongoing product development, (which) make [s] us feel [that it is difficult] to believe how they recognize the current business direction precisely and how they can run their products logically without enough foundation in the industry.

Another reason given was that angel investors evaluated the continuity exploitation of the founding team. Here, based on key points highlighted by angel investors, continuity exploitation is defined as the probability of the founding team continuously exploiting their accumulated knowledge and skills in the processes of business opportunity evaluation, and product creation and development. Specifically, all angel investors needed to validate whether the founder's accumulated knowledge could be exploited in current and future and product development plans. AngelEduCo noted:

Their CEO and CPO (chief product officer) were technical talents... From the information the CEO provided, I thought they both had at least 10,000 hours of practices [and] accumulations in the same field. Their CMO... also had some related $R \& D$ and management experience in the field... [This] was... a perfect team. Their solid foundation can be fully exploited to develop their current products in the same industry.

AngelServiCo also stated that "based on our analysis of the connection among ServiCo's founding team and its current product demonstrations, I believe their experience and background is not just an 'ever,' it is a 'future'.” In contrast, AngelImageCo highlighted: 
I found a big difference between their founders' characteristics (particularly the industrial working experience) and business activities. This may imply that this founding team's rich experience and thick accumulation associated with [the] manufacturing industry may be difficult to [transfer] into their current product designs.

\subsubsection{Venture Capitalist Scrutinizing}

At the VC financing stage, consistency across characteristic, action, and endorsement signals could help VC investors evaluate the quality and market value of a venture more effectively. All participant VC investors noted that any type of signal by itself was not enough to establish signal credibility. This meant that the ventures needed to communicate multiple types of signals consistently to VC investors. VCQTechCo highlighted that "consistency is informative about entrepreneurs' characters, their outcome actions, and their activities with external actors." VCMediCo explained that "we considered a sense of coherence among the founders' background, their business focuses (products), and [their] external affiliations as a signaling device for our investment decision making." Thus, consistency among founder's characteristic, action, and endorsement signals offered corroborating information for venture capitalists to better evaluate the ventures' market value. For example, VCRobotCo described their examination and investment decision processes for RobotCo in the following way:

When we met with the CEO and his co-founders, they provided lots of information about RobotCo... such as founding team members' backgrounds, robot arms (products) and... cooperation with a biological industry park... Most of the information and evidence they provided were interconnected together... Such corroborating information is easy for us to use jointly in evaluating RobotCo's market value and quality.

In contrast, VCSlockCo, VCShuttleCo, VCMediCo, VCChipCo, and VCInterCo used terms such as "disparate conclusions," "ambiguity and confusion," "inconsistent cues," "contradictory signals," and "incongruity" to summarize the situation in regard to their corresponding ventures' characteristic, action, and endorsement signals. Thus, they rejected their respective ventures' VC financing. For example, VCChipCo highlighted the signals of ChipCo, observing that "without continuity" of characteristic, action, and endorsement signals, it was difficult for ChipCo to create "a sense of venture identity and image."

VC investors emphasized the importance of consistency among characteristic, action, and endorsement signals for two main reasons. The first was that they evaluated a venture's continuity coping capability, defined here as the founding team's ability to cope continuously with problems and challenges during the processes of product introduction and market expansion. With TruckCo, VCTruckCo indicated that "by evaluating the connection among [the] founder team's background and products, we can check their ability to continuously develop and iterate their products." VCTruckCo also emphasized the importance of consistency between the undertaken actions and third-party affiliations, believing that "such affiliations enable... TruckCo to access... consistent and valuable benefits, such as suggestions on product iteration, which can help the venture to further iterate its products to fit the market needs." In contrast, VCShuttleCo's reason for rejection was as follows: "[ShuttleCo] is being affiliated with other famous firms, but we found those affiliations are ambiguous and irrelevant to their core business (products). Those irrelevant affiliations increase our perceived uncertainties in their future market value."

The other reason was that VC investors evaluated a venture's continuity growth capability, defined here as the venture's capability to continuously grow and achieve financial returns. VCSoftCo suggested that the consistency between founding team characteristics and current products enabled them to evaluate "the founding team's consistent capability to target [the] right market segmentation... [the] right way at the right time." Similarly, VCAICo commented that "if the entrepreneurs do not 
have consistent experience and background, they may have difficulties and challenges [in giving a] timely respon[se] to the market. They may face some market risks." Further, VC investors emphasized the importance of consistent endorsements, because these allowed them to observe the external reaction toward the market value of venture products. For instance, VCAICo explained that "AICo's cooperation with two Chinese listed firms is closely related to its core business and products, which will definitely add [to] our confidence in their products and their future market expansion." In contrast, VCShuttleCo noted:

Even though it (venture ShuttleCo) had various formal cooperation with governments, reputable firms, and prestigious angel investors, that cooperation is disconnected [from] its core business activities and products. This disconnection disrupts our evaluation [of] its market segmentation and market value because we worried... that they put too much effort [into] building irrelevant networks.

\section{DISCUSSION}

\subsection{Process Model}

Based on the case study analysis, the key findings of this research are presented and discussed in terms of how they contribute to the literature on signaling and entrepreneurial financing.

\subsubsection{Extracting}

Given that a venture normally sends multiple signals, the first step for investors in interpreting these signals is to extract the fundamental types of signals. Some signals are important during one financing stage and less important during another. At the angel financing stage, angel investors consider founding team characteristics a fundamental signal-these characteristics specifically include the founding team's educational background, industry experience, and prior joint working experience. The key mechanisms for the angel investors to consider characteristics as a fundamental signal are that they need to assess the founding team members' technological and learning capabilities, and their teamwork capability. However, at the VC financing stage, venture capitalists consider the visible actions of new ventures their fundamental signal. These actions particularly include possessing an innovation patent or patents and having evidence of new product introduction. VC investors use these action signals to evaluate the commercialization capability of the founding team and the potential market capacity of products. These findings lead to the following propositions:

Proposition 1a: An angel investors' first step is to extract characteristic signals when making a venture funding decision.

Proposition 1b: A venture capitalists' first step is to extract action signals when making a venture funding decision.

These findings resonate with the literature: a technology venture needs to craft key signals to prove its organizational legitimacy and quality, and to satisfy the expectations and requirements of critical resource providers as that venture evolves and grows (Fisher et al., 2016; Navis \& Glynn, 2011). Similarly, previous studies that have adopted a new venture legitimacy management and resource acquisition perspective have suggested that ventures must manage their organizational legitimacy judgments in response to different audiences (Fisher et al., 2017; Überbacher, 2014). This is because the assessment of organizational legitimacy is more likely to be audience dependent (Bitektine, 2011). In response to such a dynamic perspective on new venture legitimacy, this study highlights the fundamental signal among multiple signal types transmitted across different stages of new venture development. 
Although previous studies have focused on the signaling of new ventures, they have tended to consider new ventures theoretically and empirically, using the early stage as an aggregate concept to reflect angel and VC financing stages (e.g., Plummer et al., 2016). Alternatively, they emphasize a specific single stage (e.g., the angel financing stage). The present study investigates the early stages by focusing on both angel and subsequent VC financing. Although the literature highlights the important role played by multiple signal types in private equity financing, it fails to differentiate the roles played by multiple signals. This study extends the scholarship by adopting a dynamic view of signals across two important early growth stages of a venture to reveal the fundamental signal. Thus, it addresses calls to provide in-depth qualitative analysis to enhance understanding of the signaling dynamic through different stages of a venture's organizational life cycle (Fisher et al., 2016).

Studies on new venture financing have largely focused on the signaler perspective to examine how new ventures convey signals strategically to potential investors (e.g., Ko \& McKelvie, 2018). Most studies assume that receivers make rational responses to multiple types of signals (Drover et al., 2017). Some recent research has explored the receiver perspective (e.g., Carpentier \& Suret, 2015), mainly by examining the assessment criteria used by angel or VC investors, without considering the signaler perspective. However, the signaling theory literature indicates that the signaling process includes two primary actors - the signaler and the receiver - as well as the signal itself. Consequently, this study has enriched the entrepreneurial signaling literature by integrating both signaling and screening perspectives, and by investigating signaler-receiver dyads to examine how signal receivers perceive different signal types sent by signalers during the early life cycle of a technology venture (Connelly et al., 2011). The research answers the questions of which and when one type of signal may overpower others.

\subsubsection{Orchestrating}

The second insight of this study involves an investor's second step toward orchestrating the compositions of different signal types sent by new ventures. The study concludes that angel investors and VCs orchestrate different signal compositions for their overall signal evaluation. It is common for signal receivers to require additional signals to help them further understand the signaler. Specifically, angel investors orchestrate characteristic signals as the sole determinant signal, with actions as supplementary. However, besides viewing actions as an elementary signal, VCs orchestrate characteristic and endorsement signals as complementary signals to evaluate the action signal and venture quality. These findings are summarized by the following propositions:

Proposition 2a: An angel investor's second step is to orchestrate the characteristic signal as a determinant signal and the action signal as a supplementary signal when making a venture funding decision.

Proposition 2b: A VC investor's second step is to orchestrate the action signal as an elementary signal and characteristic as well as endorsement signals as complementary signals when making a venture funding decision.

These findings resonate with those of earlier studies into signal interactions. The new venture literature shows that multiple signals can be used independently or jointly in an IPO context (Hughes, 1986; Li \& McConomy, 2004). Recent studies have investigated signal interaction among multiple signals in either angel or VC financing acquisition (e.g., Ko \& McKelvie, 2018). However, these studies have investigated signal interaction between multiple signal types by focusing on a snapshot of the entire early financing process. The current work extends the literature by focusing on the screening process used by signal receivers to discuss the composition and interaction of multiple signal types across the early angel and subsequent VC financing resource acquisition stages. Specifically, it extends the literature by theorizing the ways in which different signal types supplement or complement each another across different financing stages. Most previous studies on 
entrepreneurial signaling have highlighted an isolated role for different signal types. They assume that different signals work independently and that the combined effect of multiple signal types is additive (e.g., Busenitz et al., 2005). Plummer et al. (2016) examined the signaling interaction between characteristic and endorsement signals, as well as action and endorsement signals. They argued that they were investigating signals and their interpretation through receiver sense-making. However, they still focused on the perspective of the signal sender by only analyzing the signals sent by ventures statistically, and then simply aggregating angel and VC financing as "early-stage financing." Other studies have adopted a similar approach and examined how signals may be contingent on certain factors (Pollock et al., 2010). However, the current research explores how interaction effects among different signal types vary during different early financing stages.

\subsubsection{Scrutinizing}

The third insight of this study concerns an investor's third step toward scrutinizing the consistency among characteristic, action, and endorsement signals across the angel and VC financing stages. The study finds that even though action signals are considered supplementary by angel investors, when they are observed and interpreted, they have to be consistent with the focal venture's characteristic signal to obtain angel financing. For VC financing, venture capitalists have to observe and interpret consistency between complementary characteristic and endorsement signals, and the action signal of the focal ventures. These findings lead to the following propositions:

Proposition 3a: An angel investor's third step is to scrutinize the consistency between determinant characteristic and supplementary action signals when making a venture funding decision.

Proposition 3b: A VC investor's third step is to scrutinize the consistency between elementary action, and complementary characteristic and endorsement signals when making a venture funding decision.

These findings resonate with the signal consistency literature, which has shown that signal effectiveness depends on signal consistency (Riley, 1975), and that multiple signals from different dimensions should be communicated consistently to enhance communication efficiency between signalers and receivers (Connelly et al., 2011). This study extends the literature by combining signaling and screening perspectives to investigate signal consistency among the multiple dimensions of signals as a venture evolves during the early stages of its life cycle. These findings echo those of previous studies (e.g., R. E. Miles \& Snow, 1978) by identifying different types of consistencies across angel and VC financing stages. Thus, this study further advances the literature by unpacking the underlying mechanisms of signal consistency across the angel and VC financing stages.

These six empirically developed insights are combined here to develop an intelligible process model that reflects signaling dynamics across the different financing stages, illustrating how signal perception and interpretation evolves over this cycle (see Figure 1).

\subsection{Practical Implications}

The findings lead to several practical suggestions for new ventures, and for angel and VC investors. First, the findings suggest that, as signalers, entrepreneurs should manage legitimacy assessment demands through managing signaling information across different investors as their venture evolves and grows. Different investors have various primary investment goals and objectives. For instance, at the angel financing stage, new ventures are advised to emphasize the characteristics of their top management team. At the subsequent VC financing stage, new ventures should shift their primary emphasis from characteristics to actions taken. Second, the findings provide guidance concerning how entrepreneurs can gain external financial support for their new ventures by systematically managing different types of signaling information. Entrepreneurs should consider the interactions among different signal types, rather than managing signaling information itself separately. For instance, at 
Figure 1. Investors' sense-making process model on signaling information

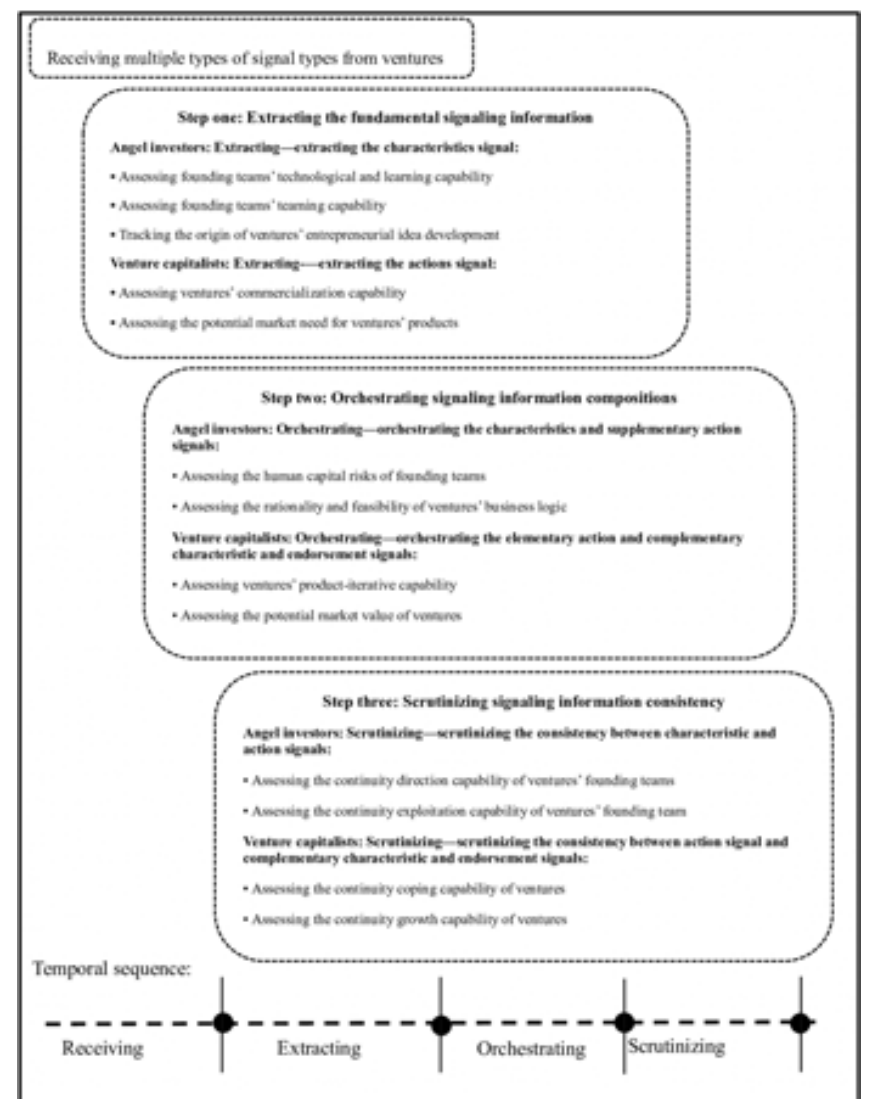

the angel financing stage, entrepreneurs must understand that characteristic signals are necessary and sufficient for them to gain an initial legitimacy, while action and endorsement signals serve unnecessary but supplementary purposes for acquiring additional legitimacy. However, at the VC financing stage, entrepreneurs should strategically manage characteristic, action, and endorsement signals as an integrative orchestration to maintain organizational legitimacy and gain funding from venture capitalists. These strategies will help new ventures gain and sustain their quality and legitimacy for different investors throughout their development stage. They may also provide investors with guidelines for evaluating potential projects more effectively.

\subsection{Limitations and Future Research Opportunities}

This study has some unavoidable shortcomings of. First, because the analysis is based on case studies of new high-technology ventures located in the Beijing Experimental Zone of Zhongguancun Council (Beijing government sector), the generalizability of the proposed conceptual framework requires testing through more substantial studies in other settings. Nonetheless, the exploratory findings of this study address current gaps in the literature on entrepreneurial financing (e.g., Connelly et al., 2011; Fisher et al., 2017; Überbacher, 2014), and contribute to improving understanding of which signal types dominate, when they dominate, and how they interact. However, the study has focused only on financing during the nascent period of a venture's life cycle, before it becomes an IPO. Future research could shift from the early to the later life cycle stages of a venture, such as the IPO stage. 
Second, most interviews were conducted at around six months after the ventures had received angel funds, and eight months after they had received VC funds; therefore, some recall bias may have occurred. The study sought to reduce this bias by using archival records, other objective data (e.g., information from venture websites), and subsequent interviews with a new venture's corresponding angel investors and venture capitalists. As is often the case with multiple case studies, further research is needed to provide more supporting data to validate the propositions and conceptual framework presented here. Thus, a quantitative study is required to test the propositions and conceptual framework and to develop more solid results.

\section{ACKNOWLEDGMENT}

We gratefully acknowledge the comments received from Prof. Jane Lu, Prof. Gracy Yang, Prof. Dilani Jayawarna and three anonymous reviewers from the 78th Annual Meeting of Management (AOM) in 2018. This research has been supported by a Research Development Fund RDF15-02-50 grant awarded by Xi' an Jiaotong-Liverpool University (XJTLU). 


\section{REFERENCES}

Aguinis, H., \& Solarino, A. M. (2019). Transparency and replicability in qualitative research: The case of interviews with elite informants. Strategic Management Journal, 40(8), 1291-1315. doi:10.1002/smj.3015

Ahlers, G. K. C., Cumming, D., Gunther, C., \& Schweizer, D. (2015). Signaling in equity crowdfunding. Entrepreneurship Theory and Practice, 39(4), 955-980. doi:10.1111/etap.12157

Becker-Blease, J., \& Sohl, J. E. (2015). New venture legitimacy: The conditions for angel investors. Small Business Economics, 45(4), 735-749. doi:10.1007/s11187-015-9668-7

Beckman, C. M., Burton, M. D., \& O'Reilly, C. (2007). Early teams: The impact of team demography on VC financing and going public. Journal of Business Venturing, 22(2), 147-173. doi:10.1016/j.jbusvent.2006.02.001

Berg, B. L., Lune, H., \& Lune, H. (2004). Qualitative research methods for the social sciences. Pearson.

Bitektine, A. (2011). Toward a theory of social judgments of organizations: The case of legitimacy, reputation, and status. Academy of Management Review, 36(1), 151-179. doi:10.5465/amr.2009.0382

Bruton, G. D., Chahine, S., \& Filatotchev, I. (2009). Founders, private equity investors, and underpricing in entrepreneurial IPOs. Entrepreneurship Theory and Practice, 33(4), 909-928. doi:10.1111/j.1540-6520.2009.00309.x

Bruton, G. D., Filatotchev, I., Chahine, S., \& Wright, M. (2010). Governance, ownership structure, and performance of IPO firms: The impact of different types of private equity investors and institutional environments. Strategic Management Journal, 31(5), 491-509. doi:10.1002/smj.822

Busenitz, L. W., Fiet, J. O., \& Moesel, D. D. (2005). Signaling in venture capitalist-new venture team funding decisions: Does it indicate long-term venture outcomes? Entrepreneurship Theory and Practice, 29(1), 1-12. doi:10.1111/j.1540-6520.2005.00066.x

Cannizzaro, A. P., \& Weiner, R. J. (2018). State ownership and transparency in foreign direct investment. Journal of International Business Studies, 49(2), 172-195. doi:10.1057/s41267-017-0117-5

Carpentier, C., \& Suret, J. M. (2015). Canadian business angel perspectives on exit: A research note. International Small Business Journal, 33(5), 582-593. doi:10.1177/0266242613516140

Cassell, C., \& Symon, G. (Eds.). (2004). Essential guide to qualitative methods in organizational research. Sage. doi:10.4135/9781446280119

Cavallo, A., Ghezzi, A., Dell'Era, C., \& Pellizzoni, E. (2019). Fostering digital entrepreneurship from startup to scaleup: The role of venture capital funds and angel groups. Technological Forecasting and Social Change, 145, 24-35. doi:10.1016/j.techfore.2019.04.022

Certo, S. T. (2003). Influencing initial public offering investors with prestige: Signaling with board structures. Academy of Management Review, 28(3), 432-446. doi:10.5465/amr.2003.10196754

Colombo, M. G., \& Grilli, L. (2009). A capital partnership: How human and venture capital affect the growth of high-tech start-ups. Strategic Change, 18(7-8), 231-239. doi:10.1002/jsc.850

Connelly, B. L., Certo, S. T., Ireland, R. D., \& Reutzel, C. R. (2011). Signaling theory: A review and assessment. Journal of Management, 37(1), 39-67. doi:10.1177/0149206310388419

Drover, W., Busenitz, L., Matusik, S., Townsend, D., Anglin, A., \& Dushnitsky, G. (2017). A review and road map of entrepreneurial equity financing research: Venture capital, corporate venture capital, angel investment, crowdfunding, and accelerators. Journal of Management, 43(6), 1820-1853. doi:10.1177/0149206317690584

Drover, W., Wood, M. S., \& Corbett, A. C. (2017). Towards a cognitive view of signaling theory: Individual attention and signal set interpretation. Journal of Management Studies, 55(2), 209-231. doi:10.1111/joms.12282

Eisenhardt, K. M. (1989). Agency theory: An assessment and review. Academy of Management Review, 14(1), 57-74. doi:10.5465/amr.1989.4279003

Eisenhardt, K. M., \& Graebner, M. (2007). Theory building from cases: Opportunities and challenges. Academy of Management Journal, 50(1), 25-32. doi:10.5465/amj.2007.24160888 
Eisenhardt, K. M., \& Schoonhoven, C. B. (1996). Organizational growth: Linking founding team, strategy, environment and growth among US semiconductor ventures, 1978-1988. Administrative Science Quarterly, 35(3), 504-529. doi:10.2307/2393315

Fisher, G., Kotha, S., \& Lahiri, A. (2016). Changing with the times: An integrated view of identity, legitimacy and new venture life cycles. Academy of Management Review, 41(3), 383-409. doi:10.5465/amr.2013.0496

Fisher, G., Kuratko, D. F., Bloodgood, J. M., \& Hornsby, J. S. (2017). Legitimate to whom? The challenge of audience diversity and new venture legitimacy. Journal of Business Venturing, 32(1), 52-71. doi:10.1016/j.jbusvent.2016.10.005

Gehman, J., Glaser, V. L., Eisenhardt, K. M., Gioia, D., Langley, A., \& Corley, K. G. (2018). Finding theorymethod fit: A comparison of three qualitative approaches to theory building. Journal of Management Inquiry, 27(3), 284-300. doi:10.1177/1056492617706029

Gimmon, E., \& Levie, J. (2010). Founder's human capital, external investment, and the survival of new hightechnology ventures. Research Policy, 39(9), 1214-1226. doi:10.1016/j.respol.2010.05.017

Gomulya, D., Kyuho, J., Lee, P. M., \& Pollock, T. G. (2019). Crossed wires: Endorsement signals and the effects of IPO firm delistings on venture capitalists' reputations. Academy of Management Journal, 62(3), 641-666. doi:10.5465/amj.2016.0796

Gomulya, D., \& Mishina, Y. (2017). Signaler credibility, signal susceptibility, and relative reliance on signals: How stakeholders change their evaluative processes after violation of expectations and rehabilitative efforts. Academy of Management Journal, 60(2), 554-583. doi:10.5465/amj.2014.1041

Hall, B. H. (2019). Is there a role for patents in the financing of new innovative firms? Industrial and Corporate Change, 28(3), 657-680. doi:10.1093/icc/dty074

Hallen, B., \& Eisenhardt, K. (2012). Catalyzing strategies and efficient tie formation: How entrepreneurial firms obtain investment ties. Academy of Management Journal, 55(1), 35-70. doi:10.5465/amj.2009.0620

Hemmert, M., Cross, A. R., Cheng, Y., Kim, J. J., Kohlbacher, F., Kotosaka, M., Waldenberger, F., \& Zheng, L. J. (2019). The distinctiveness and diversity of entrepreneurial ecosystems in China, Japan, and South Korea: An exploratory analysis. Asian Business \& Management, 18(3), 211-247. doi:10.1057/s41291-019-00070-6

Hoenig, D., \& Henkel, J. (2015). Quality signals? The role of patents, alliances and team experience in venture capital financing. Research Policy, 44(5), 1049-1064. doi:10.1016/j.respol.2014.11.011

Hsu, D. H., \& Ziedonis, R. H. (2013). Resources as dual sources of advantage: Implications for valuing entrepreneurial-firm patents. Strategic Management Journal, 34(7), 761-781. doi:10.1002/smj.2037

Hughes, P. (1986). Signalling by direct disclosure under asymmetric information. Journal of Accounting and Economics, 8(2), 119-142. doi:10.1016/0165-4101(86)90014-5

Islam, M., Fremeth, A., \& Marcus, A. (2018). Signaling by early stage startups: US government research grants and venture capital funding. Journal of Business Venturing, 33(1), 35-51. doi:10.1016/j.jbusvent.2017.10.001

Kan, A. K. S., Adegbite, E., El Omari, S., \& Abdellatif, M. (2016). On the use of qualitative comparative analysis in management. Journal of Business Research, 69(4), 1458-1463. doi:10.1016/j.jbusres.2015.10.125

Ko, E., \& McKelvie, A. (2018). Signaling for more money: The roles of founders' human capital and investor prominence in resource acquisition across different stages of firm development. Journal of Business Venturing, 33(4), 438-454. doi:10.1016/j.jbusvent.2018.03.001

Li, Y., \& Atuahene-Gima, K. (2001). Product innovation strategy and the performance of new technology ventures in China. Academy of Management Journal, 44(6), 1123-1134. doi:10.2307/3069392

Li, Y., \& McConomy, B. J. (2004). Simultaneous signaling in IPOs via management earnings forecasts and retained ownership: An empirical analysis of the substitution effect. Journal of Accounting, Auditing \& Finance, 19(1), 1-28. doi:10.1177/0148558X0401900103

McEvily, B., \& Zaheer, A. (1999). Bridging ties: A source of firm heterogeneity in competitive capabilities. Strategic Management Journal, 20(12), 1133-1156. doi:10.1002/(SICI)1097-0266(199912)20:12<1133::AID-SMJ74>3.0.CO;2-7

Miles, M. B., \& Huberman, M. A. (1994). Qualitative data analysis: An expanded sourcebook (2nd ed.). Sage. 
Miles, R. E., Snow, C. C., Meyer, A. D., \& Coleman, H. J. Jr. (1978). Organizational strategy, structure and process. Academy of Management Review, 3(3), 546-562. doi:10.5465/amr.1978.4305755 PMID:10238389

Navis, C., \& Glynn, M. A. (2011). Legitimate distinctiveness and the entrepreneurial identity: Influence on investor judgments of new venture plausibility. Academy of Management Review, 36(3), 479-499. doi:10.5465/amr.2008.0361

Pan, L., Li, X., Chen, J., \& Chen, T. (2020). Sounds novel or familiar? Entrepreneurs' framing strategy in the venture capital market. Journal of Business Venturing, 35(2), 105930. Advance online publication. doi:10.1016/j.jbusvent.2019.02.003

Park, U. D., Borah, A., \& Kotha, S. (2016). Signaling revisited: The use of signals in the market for IPOs. Strategic Management Journal, 37(11), 2362-2377. doi:10.1002/smj.2571

Petkova, A. P. (2012). From the ground up: Building new firms' reputations. In M. L. Barnett \& T. G. Pollock (Eds.), The Oxford handbook of corporate reputation (pp. 383-401). Oxford University Press. doi:10.1093/ oxfordhb/9780199596706.013.0019

Plummer, L. A., Allison, T. H., \& Connelly, B. L. (2016). Better together? Signaling interactions in new venture pursuit of initial external capital. Academy of Management Journal, 59(5), 1585-1604. doi:10.5465/amj.2013.0100

Pollock, T. G., Chen, G., Jackson, E. M., \& Hambrick, D. C. (2010). How much prestige is enough? Assessing the value of multiple types of high-status affiliates for young firms. Journal of Business Venturing, 25(1), 6-23. doi:10.1016/j.jbusvent.2009.01.003

Pollock, T. G., \& Gulati, R. (2007). Standing out from the crowd: The visibility-enhancing effects of IPOrelated signals on alliance formation by entrepreneurial firms. Strategic Organization, 5(4), 339-372. doi:10.1177/1476127007083346

Pratt, M. G. (2000). The good, the bad and the ambivalent: Managing identification among away distributors. Administrative Science Quarterly, 45(3), 456-493. doi:10.2307/2667106

Pratt, M. G. (2008). Fitting oval pegs into round holes: Tensions in evaluating and publishing qualitative research in top-tier North American journals. Organizational Research Methods, 11(3), 481-509. doi:10.1177/1094428107303349

Riley, J. G. (1975). Competitive signalling. Journal of Economic Theory, 10(2), 174-186. doi:10.1016/00220531(75)90049-6

Rindova, V. P., Willianmson, I. O., Petkova, A. P., \& Sever, J. M. (2005). Being good or being known: An empirical examination of the dimensions, antecedents, and consequences of organizational reputation. Academy of Management Journal, 48(6), 1033-1049. doi:10.5465/amj.2005.19573108

Sanders, W. M. G., \& Boivie, S. (2004). Sorting things out: Valuation of new firms in uncertain markets. Strategic Management Journal, 25(2), 167-186. doi:10.1002/smj.370

Song, L., Augustine, D., \& Yang, G. (2016). Environmental uncertainty, prospector strategy, and new venture performance: The moderating role of network capabilities. The International Entrepreneurship and Management Journal, 12(4), 1103-1126. doi:10.1007/s11365-016-0382-y

Spence, M. (1973). Job market signaling. The Quarterly Journal of Economics, 87(3), 355-379. doi: $10.2307 / 1882010$

Spence, M. (2002). Signaling in retrospect and the informational structure of markets. The American Economic Review, 92(3), 434-459. doi:10.1257/00028280260136200

Stinchcombe, A. (1965). Social structure and organizations. In J. G. March (Ed.), Handbook of organizations (pp. 142-193). Rand McNally.

Strauss, A., \& Corbin, J. (1998). Basics of qualitative research: Techniques and procedures for developing grounded theory (2nd ed.). Sage.

Stuart, T. E., Hoang, H., \& Hybels, R. C. (1999). Interorganizational endorsements and the performance of entrepreneurial ventures. Administrative Science Quarterly, 44(2), 315-349. doi:10.2307/2666998

Symon, G., Cassell, C., \& Johnson, P. (2018). Evaluative practices in qualitative management research: A critical review. International Journal of Management Reviews, 20(1), 134-154. doi:10.1111/ijmr.12120 
Tian, L., Yang, G., \& Wei, L. (2019). Speed to legal registration and nascent venture performance: A temporal dilemma for nascent entrepreneurs in an emerging economy. Journal of Small Business Management, 57(2), 476-495. doi:10.1111/jsbm.12382

Überbacher, F. (2014). Legitimation of new ventures: A review and research programme. Journal of Management Studies, 51(4), 667-698. doi:10.1111/joms.12077

Van de Ven, A. H. (2007). Engaged scholarship: A guide for organizational and social research. Oxford University Press.

Van Osnabrugge, M. (2000). A comparison of business angel and venture capitalist investment procedures: An agency theory-based analysis. Venture Capital, 2(2), 91-109. doi:10.1080/136910600295729

Vaznyte, E., \& Andries, P. (2019). Entrepreneurial orientation and start-ups' external financing. Journal of Business Venturing, 34(3), 439-458. doi:10.1016/j.jbusvent.2019.01.006

Vergne, J. P., Wernicke, G., \& Brenner, S. (2018). Signal incongruence and its consequences: A study of media disapproval and CEO overcompensation. Organization Science, 29(5), 796-817. doi:10.1287/orsc.2018.1209

Wang, T., Qureshi, I., Deeds, D., \& Ren, Y. (2019). How do technology venture signal IPO quality? A configurational approach. Journal of Business Research, 99, 105-114. doi:10.1016/j.jbusres.2019.01.039

Yang, G., \& Li, J. (2008). The development of entrepreneurship in China. Asia Pacific Journal of Management, 25(2), 335-359. doi:10.1007/s10490-007-9078-8

Yin, R. K. (1984). Case study research: Design and methods. Sage (Atlanta, Ga.).

Zott, C., \& Huy, Q. N. (2007). How entrepreneurs use symbolic management to acquire resources. Administrative Science Quarterly, 52(1), 70-105. doi:10.2189/asqu.52.1.70

Zou, H., Chen, X., \& Ghauri, P. (2010). Antecedents and consequences of new venture growth strategy: An empirical study in China. Asia Pacific Journal of Management, 27(3), 393-421. doi:10.1007/s10490-009-9157-0

Leven J. Zheng is an Assistant Professor in Innovation and Entrepreneurship at the Department of Strategic Management and Organisation (SMO), International Business School Suzhou (AACSB, EQUIS, AMBA), Xi'an Jiaotong-Liverpool University. He received his Ph.D. in Innovation and Entrepreneurship from the Management School at the University of Liverpool. His research interests focus on entrepreneurship, newly public firms, new product development, entrepreneurial founding team or senior management team as well as entrepreneurial internationalization. His research has appeared in Technovation, Journal of Business Research and Technological Forecasting \& Social Change. He uses both qualitative and quantitative research methods.

Tao Bai is currently a Lecturer in International Business at the University of Queensland, Australia. His research interests include multinational firm strategy, non-market strategy, and the intersection between, with the focus on emerging market multinationals as a field of research and practice.

Adam R. Cross is Professor of International Business and Associate Dean of Learning and Teaching, School of Humanities and Social Sciences at Xi'an Jiaotong-Liverpool University (XJTLU). Before taking on this role, Adam served for more than four years as Associate Dean for Learning and Teaching at the International Business School Suzhou (IBSS), XJTLU, during which time IBSS became the youngest business school in the world to receive accreditation by AACSB and EQUIS. Previously, Adam worked for 17 years at the Centre for International Business University of Leeds (CIBUL), UK. Adam has co-edited four books, and has contributed to more than fifty book chapters and articles in international peer-reviewed journals, including the Journal of International Business Studies, Journal of World Business, International Business Review, and Management International Review. Adam has co-edited four books, and has contributed to more than fifty book chapters and articles in international peerreviewed journals, including the Journal of International Business Studies, Journal of World Business, International Business Review, and Management International Review. His most recent textbook on International Business, co-authored with Profs. Peter Buckley and Peter Enderwick, was published by Oxford University Press in 2018. Adam's research currently focuses on the internationalization of Asian multinational firms, cross-border licensing and management of intellectual property, start-up ecosystems, technology adoption, and user innovation, with China serving as a country context for much of this work. 